\title{
Method to Correct the Velocity Variation Information of an Automatic Crash Notification System in Vehicle-to-Rigid Barrier Frontal Collisions
}

\author{
Ying Lu $\left(1,{ }^{1}\right.$ Xiaojie Ji, ${ }^{1}$ and Yu Shu ${ }^{2}$ \\ ${ }^{1}$ School of Automotive and Traffic Engineering, Jiangsu University, Zhenjiang 212013, China \\ ${ }^{2}$ Fourth People's Hospital of Zhenjiang, Zhenjiang 212000, China \\ Correspondence should be addressed to Ying Lu; luying@ujs.edu.cn
}

Received 12 January 2021; Revised 9 July 2021; Accepted 9 August 2021; Published 19 August 2021

Academic Editor: Dario Richiedei

Copyright ( 12021 Ying Lu et al. This is an open access article distributed under the Creative Commons Attribution License, which permits unrestricted use, distribution, and reproduction in any medium, provided the original work is properly cited.

Automatic crash notification systems (ACNSs) play a key role in post-accident safety. To improve the accuracy and efficiency of ACNSs, a method to correct the velocity variation information of ACNSs was established. First, after the acceleration data of sled crash tests were analysed, the factors affecting the accuracy of the velocity variation information were determined, and the influence of the discrimination threshold and acceleration curve shape on the velocity variation information was examined. Second, according to the acceleration data generated by the simulation model of a sled crash, the correlation between the accuracy of the velocity variation information and influencing factors was modelled. Third, an automatic crash notification algorithm involving a velocity variation correction function (VVCF) was proposed based on the correlation model. Finally, to verify its reliability, the improved algorithm was applied to an automatic crash notification system (ACNS) terminal. The validation results show that the ACNS terminal can accurately identify collisions and transmit accident information. Moreover, more accurate velocity variation information can be retrieved.

\section{Introduction}

Automatic crash notification systems (ACNSs) represent valuable tools to ensure post-accident safety. In the event of an accident, if the passengers in the vehicle cannot seek help themselves, the ACNS detects the vehicle collision and transmits a rescue signal and the accident information to rescue centres, thereby improving rescue efficiency and the possibility of survival [1]. ACNSs can significantly reduce the collision-to-hospital time, especially in rural areas [2]. By facilitating the timely provision of treatment, which can increase the chances of survival and alleviate the severity of injuries, ACNSs can greatly assist road accident victims [3]. It has been reported that ACNSs can likely reduce the road traffic mortality by $1.84 \%$ and $2.4-3.8 \%$ in the USA and South Australia, respectively [4].

In the ACNS design, collision identification is a critical challenge. To identify the occurrence of a collision, most existing ACNS terminals are triggered by the ignition signal of the airbag system. A major drawback of such trigger methods is that their reliability depends on the quality of the airbag system. Moreover, information regarding the occupant injury conditions and extent of damage to the vehicle cannot be directly obtained from an airbag system, which limits the scope of application of such ACNSs. Another widely used trigger method is based on the acceleration signal of the vehicle body. Such an ACNS constantly records acceleration signals. If the recorded acceleration signals or their derivatives (such as the acceleration gradient, velocity variation, or specific power) exceed the preset thresholds, the ACNS identifies that an accident has occurred and transmits the relevant information to rescue centres. Except for the acceleration peak algorithm, most of the acceleration triggering algorithms employ two levels of thresholds. The firstlevel threshold is the discrimination threshold, which distinguishes collisions from uneven road driving, emergency braking, and so on. The second-level threshold is the triggering threshold, which evaluates the severity of the 
collision. Considerable research has been performed on the setting of discrimination thresholds. By considering the vehicle emergency braking and passing of the vehicle through barricades, Xiao et al. [5] set the discrimination threshold as $-2 g\left(1 g=9.8 \mathrm{~m} / \mathrm{s}^{2}\right)$. Jia [6] compared the acceleration peaks of vehicle bodies that were generated in multiple driving experiments on uneven roads and deceleration strips and set the discrimination threshold to $-6 g$. Ge et al. [7] recommended that the discrimination threshold should be set between $-5 g$ and $-15 g$. However, because the discrimination threshold determines the starting time of the ACNS velocity variation algorithm [5], an error likely exists between the calculated and actual velocity variations, regardless of the discrimination threshold.

With the development of passive safety technologies for modern automobiles, traditional ACNSs have been succeeded by advanced automatic crash notification systems (AACNSs), which help identify whether an accident is life threatening by estimating the severity of the crash and disseminating information concerning the severity [8]. The algorithms of most of the current AACNSs are based on regression models generated by examining different accident-injury databases $[9,10]$. AACNSs commonly use abbreviated injury scale (AIS) metrics to define the severity of the occupants' injuries. Many scholars have examined the application of ACNSs in occupant injury evaluation. Schoell et al. [11] proposed an occult score combined with injurybased metrics to define a list of injuries. Kononen et al. [12] developed a multivariate logistic regression model based on the data from the National Automotive Sampling System Crashworthiness Data System (NASS-CDS) to predict the probability that a crash-involved vehicle contains one or more occupants with serious or incapacitating injuries. In this model, the independent variables included the age, belt usage, number of events, and $\Delta v$. Yoshida et al. [13] proposed four types of crash-direction-based models (that is, the frontal crash, near-side crash, far-side crash, and rear-end crash models) by using a logistic regression modelling technique and accident data from a Japanese statistical database. Stitzel et al. [14] proposed an occupant transportation decision algorithm (OTDA). This algorithm relied mainly on vehicle remote sensing technology to collect the vehicle data to predict occupant injuries and formulate the optimal transportation scheme for the injured occupants. Pal et al. [15] introduced two additional variables-intrusion magnitude and maximum deformation location-to Kononen's model to build a logistic regression model to improve the accuracy of the AACNS. Lu et al. [16] developed an injury prediction model where the velocity variation was the independent variable. The dependent variable was the probability of the driver's max abbreviated injury scale reaching or exceeding Level 3 (denoted by MAIS3+). The authors noted that as the velocity variation increased, the probability of the driver's injury level reaching MAIS3+ increased. Therefore, the accuracy of the velocity variation directly affects the prediction accuracy of the driver's injury level.

The abovementioned studies indicated that regardless of how the injuries are defined, the velocity variation (expressed by $\Delta v$ ) is one of the most important pieces of accident information because it serves as the basis for the evaluation of occupant injuries. The accuracy of the velocity variation determination directly affects the evaluation accuracy of the occupant's injury level. In addition, the velocity variation also plays an important role in traffic accident reconstruction. Specifically, this variation can be considered to calculate the collision velocities of the vehicles involved in a collision $[17,18]$. After the initial velocity of the vehicle is determined, accident reproduction and the division of responsibility can be realized according to the relevant judicial expertise [19-21]. Therefore, it is necessary to conduct an indepth study on the methods to accurately determine the velocity variation information.

This study was aimed at developing a method to correct the velocity variation to increase the accuracy and efficiency of ACNSs. Due to the complexity of automotive body structures and materials used in vehicles, the collision accelerations of different vehicles are quite different from each other even if the initial collision speeds are identical [22]. Sled test systems have been used by automotive industries to develop safety systems. The collision acceleration curves of different sled test systems are different from one another. According to the Federal Motor Vehicle Safety Standard (FMVSS) in the U.S.A, as long as the accelerations are within the range of the upper and lower bounds recommended by the FMVSS, the sled systems are reliable [23]. Since definite expressions for collision accelerations in terms of the initial collision speeds do not exist, it is difficult to derive a theoretical model to correct the error caused by the discrimination threshold in the ACNS. The remaining paper is organized as follows. Section 2 describes the analysis of the factors that affect the accuracy of velocity variation by considering the acceleration data generated in sled tests. Section 3 describes the modelling of the correlation between the velocity variation error and influencing factors. Section 4 introduces the ACNS velocity variation algorithm developed based on the correlation model. Section 5 details the reliability of the novel algorithm applied to an ACNS terminal. Section 6 discusses the sensitivity of the algorithm and its applicability in the case of head-to-head collision. The final section presents the concluding remarks and discusses the scope for future research.

\section{Factors Affecting the Accuracy of Velocity Variation}

2.1. Discrimination Threshold. Typically, to reduce the probability of false triggering on uneven roads, a relatively large absolute value of the discrimination threshold value is set. However, because the ACNS begins to calculate the velocity variation only when the intensity of the acceleration signal collected by the acceleration sensor is larger than the discrimination threshold, the selected discrimination threshold definitely affects the calculated velocity variation.

The acceleration data in the vehicle collision process are the basis for analysing the relationship between the discrimination threshold and velocity variation. However, the cost of real vehicle testing is high, and only a limited set of acceleration data can be obtained in one real vehicle test. The 
amount of data cannot meet the research needs. A sled test is considerably more economical than a real vehicle crash test and can be implemented multiple times. Therefore, in this study, sled tests were adopted instead of real vehicle crash tests. The DAPG-QJ-MNPZ sled impact system was used in this study. The main components of the system are as follows:

(1) The sled, as shown in Figure 1, can withstand a maximum load mass of $1000 \mathrm{~kg}$. In this test, the total mass of the sled is $400 \mathrm{~kg}$. The speed range of the sled is $8-80 \mathrm{~km} / \mathrm{h}$. The accuracy is $\pm 1 \%$.

(2) The sled track, as shown in Figure 2, travels along the track during the entire test, and its length is $55 \mathrm{~m}$.

(3) The traction system, as shown in Figure 3, consists of mainly a swivel hub and hydraulic control cabinet. The objective of this system is to collide at a specified speed through a steel rope traction sled.

(4) The collision system, as shown in Figure 4, consists of an olive head and energy-absorbing device. The olive head is installed at the front of the sled. The energyabsorbing device is installed in parallel on a fixed barrier. The energy-absorbing device is a sleeve made of polyurethane energy-absorbing material and is used to absorb the energy generated when the sled collides. Generally, when the total mass of the sled is increased by $200 \mathrm{~kg}$, an energy absorption device is added. Because the total weight of the sled in the test is $400 \mathrm{~kg}, 2$ energy-absorbing devices are required. The size of the energy-absorbing device and amount of the corresponding energy-absorbing materials must be adjusted to ensure that the acceleration curve during the collision is within the range specified in the Chinese National Standard GB141662013, which is similar to the requirement in FMVSS [24].

(5) The main console is shown in Figure 5. The operation interface of the main console is shown in Figure 6. The main console is used to set up the entire crash test to control the movement of the console car. The system parameters, such as the test speed, acceleration time, and speed stabilization time, are set through the main console of the sled collision test system. In the test, linear acceleration is selected, and the initial velocity is $15 \mathrm{~km} / \mathrm{h}$. The acceleration and speed stabilization durations are set as $3 \mathrm{~s}$ and $10 \mathrm{~s}$, respectively. After the parameter setting is completed, the sled test system is implemented to conduct the crash test.

In addition, the sled crash test system has its own acceleration acquisition device, which can accurately acquire and save the acceleration data of the sled in the forward direction in the event of a large collision.

Based on the acceleration data obtained from the sled test, the relationship between the discrimination threshold and accuracy of velocity variation can be determined. The actual velocity variation in the collision process can be expressed as

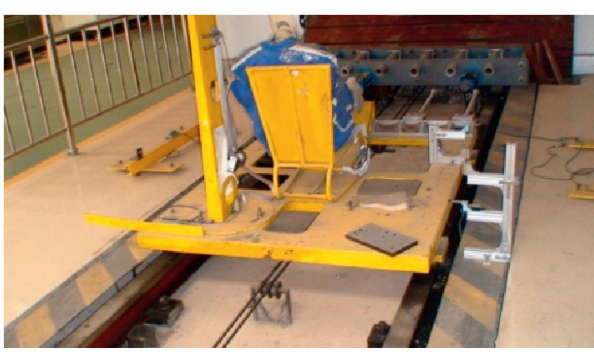

Figure 1: Test sled.

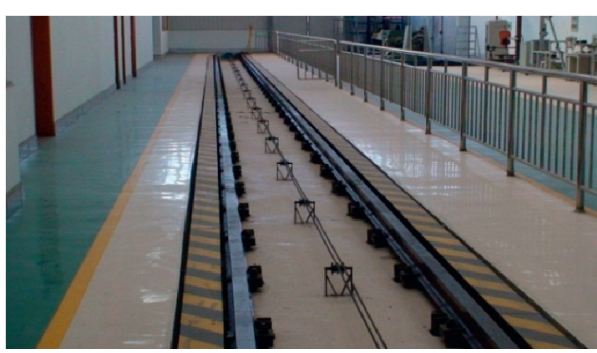

Figure 2: Sled track.

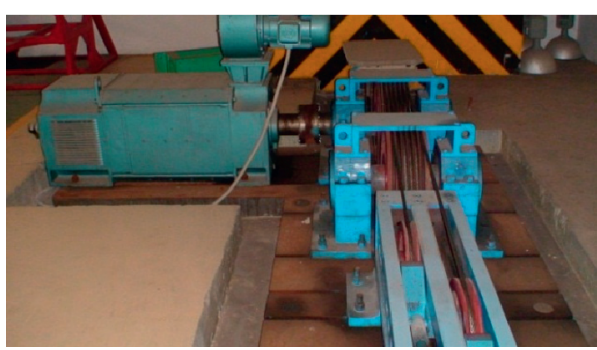

Figure 3: Traction system.

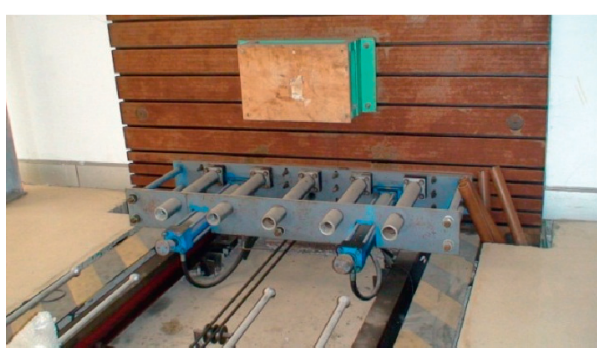

Figure 4: Collision system.

$$
\Delta v=\int_{t_{0}}^{t_{\mathrm{p}}} a(t) \mathrm{d} t
$$

where $\Delta v$ is the velocity variation in the process of collision; $t_{0}$ is the start of collision; $t_{\mathrm{p}}$ is the end of collision, that is, the time at which the acceleration of the vehicle returns to $0 g$; $t$ is the current moment; and $a(t)$ is the acceleration of the vehicle corresponding to the current moment.

Due to the difficulty in determining the exact start of the collision, the ACNS commonly adopts the time at which the acceleration of the vehicle reaches the discrimination threshold for the first time to substitute $t_{0}$. In this case, 


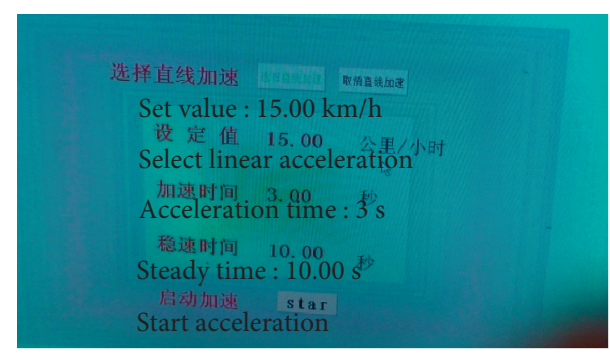

Figure 5: Main console.

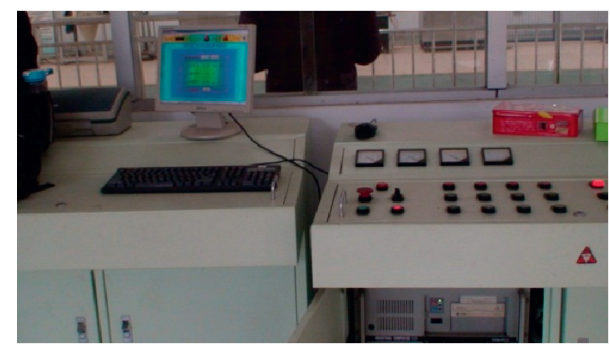

FIGURE 6: Main console operation interface.

$$
\Delta v_{A C N}=\int_{t_{d t}}^{t_{\mathrm{p}}} a(t) \mathrm{d} t,
$$

where $\Delta v_{\mathrm{ACN}}$ is the velocity variation calculated by the ACNS, and $t_{d t}$ is the time at which the acceleration of the vehicle reaches the discrimination threshold for the first time.

As shown in Figure 7, when the discrimination threshold is $-2 g$, the starting time of the collision determined by the ACNS is $t_{d t}$, and the calculated velocity variation in the collision process is the acceleration integral value from $t_{d t}$ to tp. When the discrimination threshold is $-2 g$, the starting time of the collision determined by the ACNS is $t_{d t}^{\prime}$. At this time, the calculated velocity variation in the collision process is the acceleration integral value from $t_{d t}^{\prime}$ to tp. Clearly, under different discrimination thresholds, the velocity variation calculated by the ACNS is different since the threshold cannot be $0 g$. Consequently, an error in the calculation is expected to appear for all threshold values.

We consider the velocity variation error to represent the accuracy of the velocity variation, which is defined as the difference between $\Delta v$ and $\Delta v_{\mathrm{ACN}}$. Let $E$ be the velocity variation error:

$$
E=\Delta v-\Delta v_{\mathrm{ACN}}
$$

According to this analysis, a smaller absolute value of the discrimination threshold corresponds to a smaller $E$. This finding indicates that a correlation exists between the discrimination threshold and $E$.

2.2. Acceleration Curve Shape. In addition to the discrimination threshold, the shape of the collision acceleration curve directly affects the velocity variation. When a car collides with another object at different initial velocities, the acceleration curves are different from one another. For

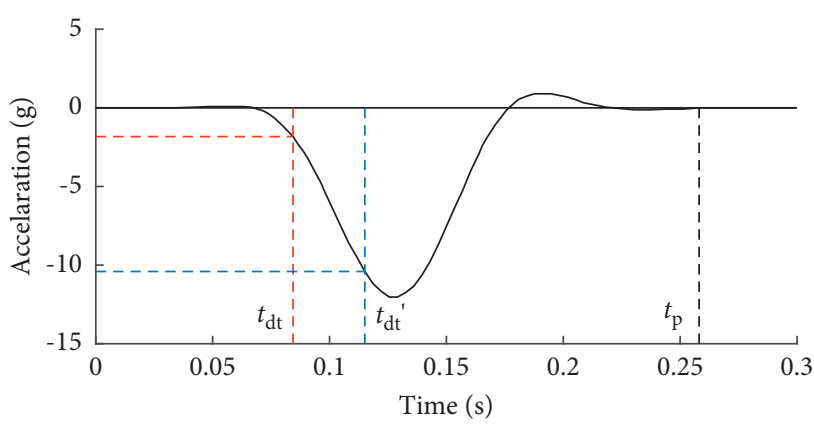

FIGURE 7: Function of discrimination threshold.

different shapes of acceleration curves, the integral values of the acceleration data (that is, the velocity variation) are different. Several characteristic variables of acceleration can be used to describe the shape of the acceleration curve, including the acceleration peak, acceleration slope, and velocity variation in a certain window width. The acceleration peak is the maximum value of the reverse acceleration during the collision of the vehicle. The acceleration slope is the derivative of the acceleration data during the collision. The velocity variation in a certain window width is the integral value calculated in the given window width [5]. Because it is difficult to directly observe the relationship among these variables, the discrimination threshold, and $E$, we perform regression analyses on these variables to identify the optimal correlation model.

\section{Correlation Model between the Velocity Variation Error and Influencing Factors}

3.1. Sled Crash Simulation Model. To establish the regression equation between the velocity variation error and influencing factors, a considerable amount of collision acceleration data is required. Commonly, a regression can be obtained using real data. However, considering the high cost and effort associated with conducting multiple real vehicle tests and sled tests, simulation models are widely utilized in the current vehicle crash studies to generate data for further analysis [23]. Simulation software is needed to simulate the vehicle collision process. The LS-DYNA simulation software has been widely used in the research field of vehicle collisions. Consequently, in this paper, we used LS-DYNA R7.0.0 to establish a sled crash simulation model and simulate the sled test process. The components of the sled crash simulation model were consistent with those of the DAPG-QJMNPZ sled impact system. To ensure that the simulated data were similar to the sled test data, the dimensions of the sled were measured to build a three-dimensional simulation model, and the materials for each part were determined to set the collision conditions. The specific parameters of this sled impact system were as follows:

(1) The sled was composed mainly of a flat plate with a length and width of $1800 \mathrm{~mm}$ and $20 \mathrm{~mm}$, respectively. The mass of the sled was $400 \mathrm{~kg}$, and the material was 45 steel. 
(2) The diameter of the olive head in front of the sled was $46.4 \mathrm{~mm}$, and the material was 45 steel.

(3) The olive head and plate were connected by a strut. The length and diameter were $645 \mathrm{~mm}$ and $27 \mathrm{~mm}$, respectively. The material was 45 steel.

(4) The polyurethane energy absorber tube had an outer diameter of $59.1 \mathrm{~mm}$ and an inner hole with a circular platform structure. The inner diameter of the tube was $44.5 \mathrm{~mm}$ near the impact end and $21.8 \mathrm{~mm}$ away from the impact end. The tube was made of a polyurethane composite material [25].

(5) The length and outer diameter of the guide tube were $655.0 \mathrm{~mm}$ and $80.0 \mathrm{~mm}$, respectively. The material was 45 steel. The polyurethane energy-absorbing tube was installed in the guide tube.

(6) The steel barrier in which the guide tube was installed was $1800 \mathrm{~mm}$ long, $20 \mathrm{~mm}$ wide, and $1000 \mathrm{~mm}$ high. The material was 45 steel.

The final sled crash simulation model is shown in Figure 8.

Before using the simulation model to generate data for the subsequent analysis, we compared the simulation results with those obtained experimentally to validate the simulation model. Figure 9 shows the crash acceleration curves pertaining to the simulation and test results for $30 \mathrm{~km} / \mathrm{h}$ after filtering the disturbing acceleration. In general, vehicle vibrations and electromagnetic interference generate noise and other unwanted signal components in the inertial signal samples. Therefore, it is necessary to select the appropriate signal filtering technique to minimize the noise and maximize the signal [26]. The acceleration data of the simulation test were filtered with a CFC60 SAE filter. The acceleration data of the sled test were measured at a sampling rate of $1000 \mathrm{~Hz}$ and filtered with a CFC60 filter [27]. In this paper, the $x$-axis pointed forward from the sled or vehicle. When a collision did not occur, the acceleration was 0 . After the collision was initiated, the acceleration direction was along the negative $x$-axis, and thus, the acceleration varied from 0 to a less-than-zero value. After the peak occurred, the collision ended, and the sled began to return. During the rebound, the acceleration direction was consistent with the positive $x$-axis, so the acceleration value at the end of the rebound was greater than 0 . After the rebound, the acceleration decreased and finally became 0 . The tested sled reached a peak acceleration of $-24.9 \mathrm{~g}$ at $65 \mathrm{~ms}$ and stopped moving at $150 \mathrm{~ms}$. The simulated sled reached a peak acceleration of $-19.5 \mathrm{~g}$ at $63 \mathrm{~ms}$ and stopped moving at $151 \mathrm{~ms}$. Considering the curve track, peak acceleration, occurrence time, and stop time, the simulated and tested curves were considered to be in agreement. These findings indicated that the sled collision simulation can accurately reproduce the sled collision process, which demonstrates the model accuracy. Therefore, the proposed model can be used for subsequent research. As long as the initial velocity of the sled varies, the collision acceleration data of the sled at different initial velocities can be obtained, which represents the data basis for establishing the regression model.

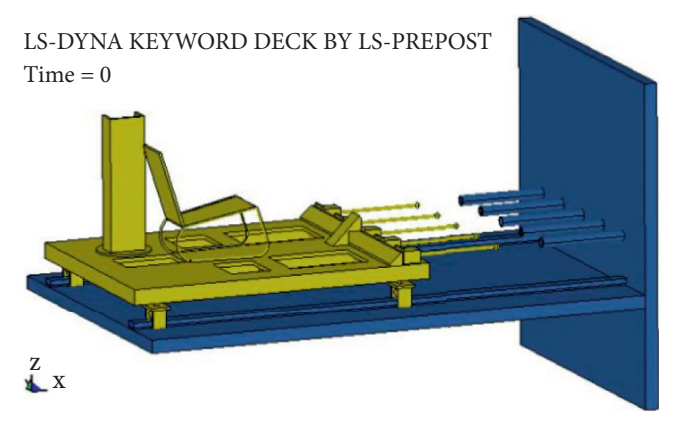

FiguRE 8: Sled crash simulation model.

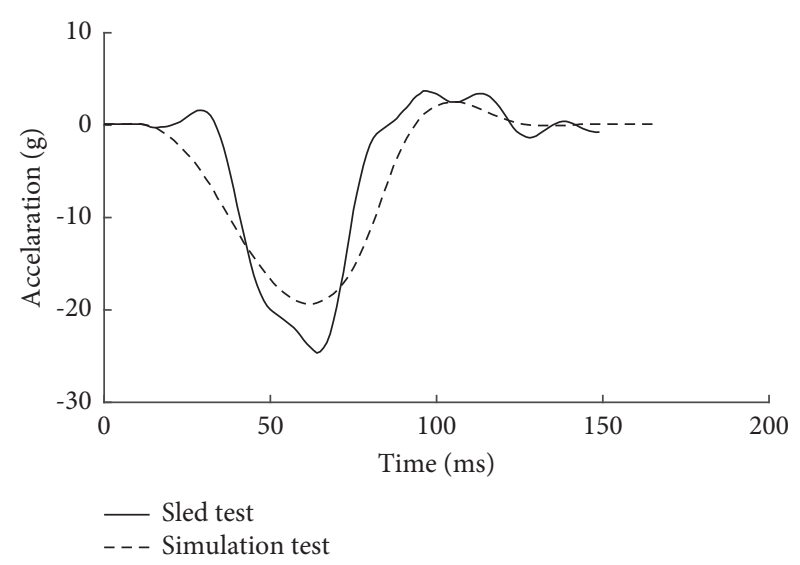

FIGURE 9: Acceleration curve of the simulation and real sled crash test after filtering at $30 \mathrm{~km} / \mathrm{h}$.

3.2. Regression Analysis. After the sled crash simulation model is developed, it is necessary to perform a regression analysis for the velocity variation error, discrimination threshold, and acceleration curve shape. Note that the shape of the acceleration curve can be described by three kinds of variables-an acceleration peak, an acceleration slope, and a velocity variation in a certain window width. Hence, these variables should not be incorporated in the same regression equation. Only one of the three variables and the discrimination threshold value can be selected as the independent variables, with the velocity variation error used as the dependent variable to construct the regression equation. To identify the most obvious correlation relationship among these three kinds of variables with the velocity variation error, we conducted a regression analysis of the velocity variation, discrimination threshold, and the three variables based on the verified simulation data $[28,29]$.

3.2.1. Acceleration Peak as an Independent Variable. The acceleration peak is the maximum value of the reverse acceleration during the collision of a vehicle. According to the acceleration data obtained by an LS-DYNA simulation, if the initial velocity of vehicle collision increases, the acceleration peak increases, which indicates that the acceleration peak and initial velocity of collision are positively correlated. Since the initial velocity of collision directly affects the shape of the collision curve and thus the velocity variation 
calculated by the ACNS, the acceleration peak and $E$ are likely correlated.

We conducted 12 sled impact simulation tests with different initial speeds to generate multiple acceleration curves and recorded the corresponding acceleration peaks associated with $E$ for different $D$. In general, to analyse the relationship among the acceleration peak, discrimination threshold, and $E$, it is necessary to determine whether the relationship between these variables is linear or nonlinear. A scatter plot can clearly depict the data points of multiple variables, and the correlations among these variables can be identified by observing the distribution of the data points. Hence, we used MATLAB R2015a to draw a scatter plot to demonstrate the distribution of data points, where $D$ denotes the discrimination threshold and $M$ denotes the acceleration peak, as shown in Figure 10.

Figure 10 shows that the data points form a plane in three-dimensional space with two independent variables and one dependent variable, so multiple linear regression analysis was used in this work. Many regression models are available, such as the linear model, quadratic model, exponential model, power model, and logarithmic model. The least-squares method finds the best function to match the data by minimizing the sum of squares of the errors. This method can be used to obtain unknown data, and the sum of squares of the errors between the obtained data and actual data is minimized. We used the least-squares method to perform multiple regression analysis, with $E$ regarded as the dependent variable and $D$ and $M$ considered as the independent variables.

We implemented the linear regression process in SPSS 16.0. The default $95 \%$ confidence interval was selected. The statistics of the regression model are shown in Table 1, and the coefficients and their significance are shown in Table 2.

Tables 1 and 2 show that the $R$ square of the entire model is 0.560 . All the coefficients, except for the constant, are statistically significant at $P<0.05$.

Figure 10 demonstrates that a curvilinear relationship exists among $E, D$, and $M$. Since the relationship between $E$, $M$, and $D$ is curvilinear, the quadratic model was chosen here. In addition, because the quadratic model is relatively simple, it can be easily programmed in the ACNS. The quadratic model for $E$ in terms of $D$ and $M$ can be expressed as

$$
E=\left(b_{1}+b_{2} D+b_{3} M+b_{4} D^{2}+b_{5} M^{2}+b_{6} D M\right)+\varepsilon,
$$

where $b_{1}$ is the coefficient of the constant term; $b_{2}$ and $b_{3}$ are the coefficients of the primary terms of $D$ and $M$, respectively; $b_{4}$ and $b_{5}$ are the coefficients of the square terms of $D$ and $M$, respectively; $b_{6}$ is the coefficient of the interaction term of $D$ and $M$; and $\varepsilon$ is the regression error. Let $\widehat{E}$ be the estimated value of $E$, which can be defined as

$$
\hat{E}=b_{1}+b_{2} D+b_{3} M+b_{4} D^{2}+b_{5} M^{2}+b_{6} D M .
$$

We implemented the regression process in SPSS 16.0. The default $95 \%$ confidence interval was selected. The statistics of the quadratic model are shown in Table 3, and the coefficients and their significance are shown in Table 4.

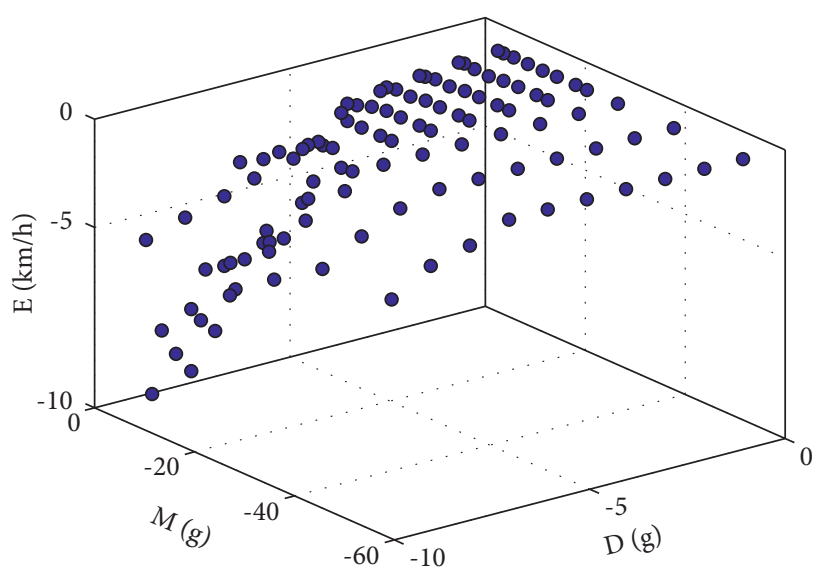

FIGURE 10: Velocity variation error-acceleration peak-discrimination threshold relationship diagram.

TABLE 1: Statistics of the linear model (acceleration peak involved).

\begin{tabular}{lc}
\hline Name & Value \\
\hline$R$ square & 0.560 \\
Adjusted $R$ square & 0.552 \\
Standard error of regression & 1.046 \\
Sum of residuals & 117.179 \\
$F$ statistic & 68.026 \\
$P$ & 0.000 \\
\hline
\end{tabular}

TABLE 2: Significance of coefficients (acceleration peak involved).

\begin{tabular}{lccc}
\hline Variable & Coefficient & $T$ statistic & $P$ \\
\hline$C^{*}$ & 0.248 & 0.886 & 0.378 \\
$D$ & 0.370 & 10.642 & 0.000 \\
$M$ & -0.033 & -4.776 & 0.000 \\
\hline
\end{tabular}

${ }^{*} \mathrm{C}$ denotes the constant term.

TABLE 3: Statistics of the quadratic model (acceleration peaks included).

\begin{tabular}{lc}
\hline Name & Value \\
\hline$R$ square & 0.792 \\
Adjusted $R$ square & 0.782 \\
Standard error of regression & 0.730 \\
Sum of residuals & 55.476 \\
$F$ statistic & 78.999 \\
$P$ & 0.000 \\
\hline
\end{tabular}

The comparison of Tables 1 and 3 indicates that the quadratic model exhibits a higher performance than the linear model, with a greater adjusted $R$ square and lower standard error of regression. Tables 3 and 4 indicate that the $R$ square of the entire model is 0.792 , and the $P$ value is 0 , which is less than $5 \%$ of the significance level, thereby indicating that the model is reliable. The $P$ values corresponding to $D$ and $M$ are 0.256 and 0.124 , respectively, both of which are greater than the $5 \%$ significance level. The $P$ values corresponding to $D^{2}, D M$, and $M^{2}$ are 0,0 , and 0.002 , respectively, which are less than $5 \%$ of the significance level. The absolute values of the corresponding $T$ statistics of $D^{2}$, $D M$, and $M^{2}$ are greater than 2 , indicating that the 
TABLE 4: Significance of coefficients (acceleration peaks included).

\begin{tabular}{lccccc}
\hline & & & \multicolumn{3}{c}{$\begin{array}{c}\text { Collinearity } \\
\text { statistics }\end{array}$} \\
Variable & Coefficient $^{*}$ & $T$ statistic & $P$ & \multicolumn{3}{c}{ Tolerance } & VIF \\
\hline$C$ & & & & \\
$D$ & -0.572 & -1.234 & 0.220 & & \\
$M$ & -0.133 & -1.142 & 0.256 & 0.043 & 23.090 \\
$D^{2}$ & -0.037 & -1.549 & 0.124 & 0.040 & 25.119 \\
$M^{2}$ & -0.073 & -7.610 & 0.000 & 0.050 & 19.906 \\
DM & -0.001 & -3.135 & 0.002 & 0.047 & 21.453 \\
\hline
\end{tabular}

${ }^{*}$ These coefficients correspond to $b_{1}, b_{2}, b_{3}, b_{4}, b_{5}$, and $b_{6}$ sequentially.

significance test is passed. In regression analyses, it is necessary to test whether multiple correlation problems exist among the variables. The tolerance and variance inflation factor (VIF) test was performed in this study. If the tolerance value is greater than 0.1 and the VIF value is less than 10 , no multicollinearity is considered to exist among the variables. According to Table 4, the tolerance values of $D, M, D^{2}$, and $M^{2}$ are less than 0.1, and their VIF values are greater than 10. Thus, significant multicollinearity likely occurs among these independent variables, except for $D M$.

We excluded the variables that were not statistically significant at the $5 \%$ significance level (that is, $D$ and $M$ ) to adjust the initial regression model. The statistics of the adjusted quadratic model are shown in Table 5. The coefficients and their significance values are shown in Table 6.

According to the two tables, the $R$ square of this model is 0.785 , and the $P$ value is 0 , which is less than $5 \%$ of the significance level, indicating that the model is reliable. The $P$ values corresponding to the independent variables are less than $5 \%$ of the significance level, and the absolute values of the $T$ statistic are greater than 2 , which indicates that the significance test is passed. Because the tolerance values of the independent variables are greater than 0.1 and their VIF values are less than 10 , no significant multicollinearity is considered to exist among the independent variables. The adjusted regression equation is expressed as equation (6), and its three-dimensional graph is shown in Figure 11.

$$
\stackrel{\hat{E}}{=} 0.232-0.065 D^{2}-0.0005 M^{2}+0.013 D M .
$$

3.2.2. Mean Absolute Values of the Acceleration Slopes as an Independent Variable. The acceleration slope describes the slope corresponding to each data point on the acceleration curve, which indicates the speed of change in acceleration. Let $J(t)$ be the acceleration slope. In this case,

$$
J(t)=\frac{a(t)-a(t-\Delta t)}{\Delta t},
$$

where $a(t-\Delta t)$ is the acceleration of the previous time relative to the current moment, and $\Delta t$ is the time interval between two acceleration signals.

For a certain initial velocity of the collision, $J(t)$ is clearly not unique. The data amount $J(t)$ is determined by the number of data points included in the acceleration curve. Hence, a one-to-one correspondence does not exist between
TABLE 5: Statistics of the adjusted quadratic models (acceleration peaks included).

\begin{tabular}{lc}
\hline Name & Value \\
\hline$R$ square & 0.785 \\
Adjusted $R$ square & 0.779 \\
Standard error of regression & 0.73471 \\
Sum of residuals & 57.219 \\
$F$ statistic & 129.031 \\
$P$ & 0.000 \\
\hline
\end{tabular}

TABle 6: Significance of coefficients of the adjusted model (acceleration peaks included).

\begin{tabular}{lccccc}
\hline & & & \multicolumn{3}{c}{ Collinearity } \\
Variable & Coefficient & $T$ statistic & $P$ & Tolerance & VIF \\
\hline$C$ & & & & & \\
& & & & & \\
$D^{2}$ & -0.232 & 1.832 & 0.070 & & \\
$M^{2}$ & -0.0005 & -16.571 & 0.000 & 0.306 & 3.272 \\
$D M$ & 0.013 & 8.961 & 0.000 & 0.170 & 5.899 \\
\hline
\end{tabular}

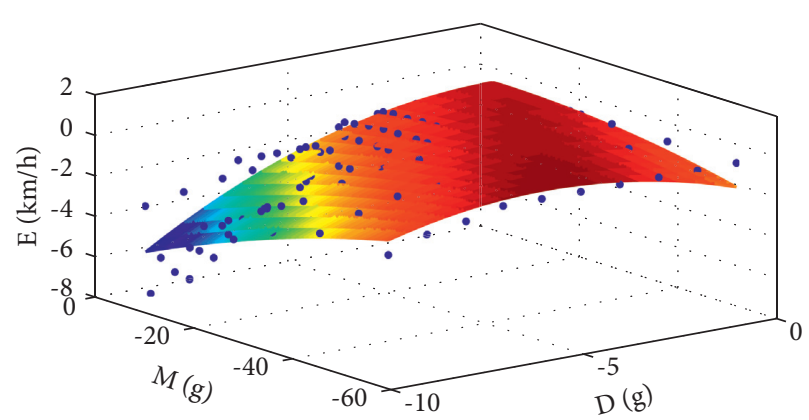

FiguRE 11: Three-dimensional graph of the velocity variation erroracceleration peak-discrimination threshold regression equation.

$J(t)$ and $E$. In addition, because the acceleration slope can be positive and negative, the mean acceleration slope is close to 0 . Consequently, to establish the relationship between the acceleration slope and velocity variation error, we used the mean absolute values of the acceleration slope as the independent variables rather than the mean acceleration slope. Let $J$ denote the mean of the absolute values of the acceleration slope, defined as

$$
J=\frac{1}{N} \sum_{t}|J(t)|
$$

where $N$ is the number of sample points in the time domain.

Note that for each acceleration curve, there exists a corresponding $J$. In this way, a one-to-one correspondence can be achieved between the variables. We calculated the corresponding $J$ of the acceleration curves associated with different $D$. Then, we constructed a scatter plot involving $D$, $J$, and $E$, as shown in Figure 12.

We used the least-squares method to perform a multiple regression analysis, where $E$ was regarded as the dependent variable and $D$ and $J$ were used as the independent variables. We implemented the linear regression process in SPSS 16.0. The default $95 \%$ confidence interval was selected. The 


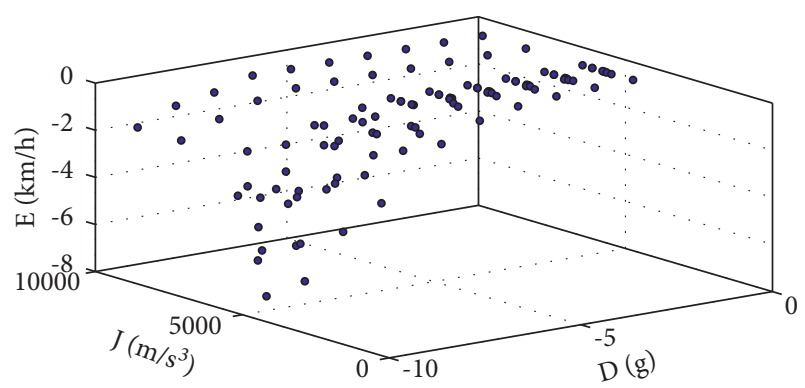

FIGURE 12: Velocity variation error-mean absolute acceleration slope-discrimination threshold relationship diagram.

statistics of the regression model are shown in Table 7, and the coefficients and their significance are shown in Table 8.

Tables 7 and 8 show that the $R$ square of the entire model is 0.523 . All the coefficients are statistically significant at $P<0.05$, except for the constant term.

In addition, we adopted a quadratic model for the regression analysis. The expression of the quadratic model was similar to equation (5), albeit with $J$ substituted for $M$. These data were input to SPSS 16.0. The default 95\% confidence interval was selected. The statistics of the quadratic model are shown in Table 9, and the coefficients and their significances are shown in Table 10.

The comparison of Tables 7 and 9 indicates that the quadratic model seems to perform better than the linear model does, with a greater adjusted $R$ square and lower standard error of regression. The data in the aforementioned tables show that the $R$ square of this model is 0.703 , and the $P$ value is 0 , which is less than $5 \%$ of the significance level, indicating that the model is reliable. The $P$ value corresponding to $D^{2}$ and DJ is 0 , less than $5 \%$ of the significance level, and the absolute value of the $T$ statistic is greater than 2 , indicating that the significance test is passed. However, the $P$ value corresponding to $J^{2}$ is 0.132 , which is greater than the significance level, and the absolute value of the corresponding $T$ statistic is less than 2 . The $P$ values corresponding to $D$ and $J$ are 0.840 and 0.299 , respectively, which are greater than the $5 \%$ significance level. The absolute value of the corresponding $T$ statistic of $D$ and $J$ is less than 2 . Therefore, a linear relationship does not exist between the two independent variables and dependent variable, and the nonlinear relationship is not significant. Because the tolerance values of $D, J, D^{2}, J^{2}$, and $D J$ are less than 0.1 and their VIF values are greater than 10 , significant multicollinearity is considered to exist among the independent variables.

We excluded variables that were not statistically significant at the $5 \%$ significance level (that is, $D$ and $J$ ) to adjust the intimal regression model. The statistics of the adjusted quadratic model are shown in Table 11, and the coefficients and their significances are shown in Table 12.

The two aforementioned tables indicate that the $R$ square of this adjusted model is 0.699 , and the $P$ value is 0 , which is less than $5 \%$ of the significance level, indicating that the model is reliable. The $P$ values corresponding to $D^{2}$, $J^{2}$, and $D J$ are less than $5 \%$ of the significance level, and the absolute values of the $T$ statistic are greater than 2 , which
TABLE 7: Statistics of the linear model (mean absolute values of the acceleration slopes).

\begin{tabular}{lc}
\hline Name & Value \\
\hline$R$ square & 0.523 \\
Adjusted $R$ square & 0.515 \\
Standard error of regression & 1.089 \\
Sum of residuals & 126.849 \\
$F$ statistic & 58.761 \\
$P$ & 0.000 \\
\hline
\end{tabular}

TABle 8: Significance of coefficients (mean absolute values of the acceleration slopes).

\begin{tabular}{lccc}
\hline Variable & Coefficient & $T$ statistic & $P$ \\
\hline$C$ & -0.250 & -0.573 & 0.568 \\
$D$ & 0.370 & 10.228 & 0.000 \\
$J$ & 0.000 & 3.593 & 0.000 \\
\hline
\end{tabular}

TABLE 9: Statistics of quadratic models (mean absolute values of the acceleration slopes).

\begin{tabular}{lc}
\hline Name & Value \\
\hline$R$ square & 0.703 \\
Adjusted $R$ square & 0.688 \\
Standard error of regression & 0.872 \\
Sum of residuals & 79.115 \\
$F$ statistic & 49.179 \\
$P$ & 0.000 \\
\hline
\end{tabular}

TABLE 10: Significance of coefficients (mean absolute values of the acceleration slopes).

\begin{tabular}{|c|c|c|c|c|c|}
\hline \multirow[t]{2}{*}{ Variable } & \multirow[t]{2}{*}{ Coefficient } & \multirow[t]{2}{*}{$T$ statistic } & \multirow[t]{2}{*}{$P$} & \multicolumn{2}{|c|}{$\begin{array}{c}\text { Collinearity } \\
\text { statistics }\end{array}$} \\
\hline & & & & Tolerance & VIF \\
\hline$C$ & -1.585 & -0.947 & 0.346 & & \\
\hline$D$ & 0.034 & 0.202 & 0.840 & 0.030 & 32.972 \\
\hline$J$ & 0.001 & 1.044 & 0.299 & 0.011 & 87.082 \\
\hline$D^{2}$ & -0.073 & -6.372 & 0.000 & 0.050 & 19.906 \\
\hline$J^{2}$ & $-6.70 E-8$ & -1.518 & 0.132 & 0.012 & 83.415 \\
\hline DJ & $-9.20 E-5$ & -4.454 & 0.000 & 0.056 & 17.732 \\
\hline
\end{tabular}

TABLE 11: Statistics of the adjusted quadratic models (mean velocity variation in a certain window width).

\begin{tabular}{lc}
\hline Name & Value \\
\hline$R$ square & 0.699 \\
Adjusted $R$ square & 0.691 \\
Standard error of regression & 0.869 \\
Sum of residuals & 80.032 \\
$F$ statistic & 82.180 \\
$P$ & 0.000 \\
\hline
\end{tabular}

indicates that the significance test is passed. The tolerance values of $D^{2}$ and $J^{2}$ are greater than 0.1 , and the VIF values are less than 10 . No significant multicollinearity is considered to exist among the independent variables except for $D J$. The adjusted regression equation is expressed as equation (9), and its three-dimensional graph is shown in Figure 13. 


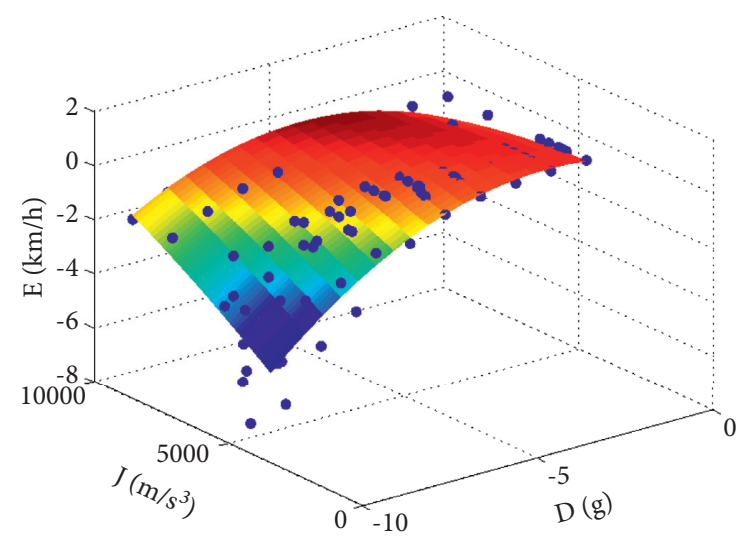

FIGURE 13: Three-dimensional graph of velocity variation errormean absolute acceleration slope-discrimination threshold regression equation.

TABLE 12: Significance of coefficients of the adjusted model (mean velocity variation in a certain window width).

\begin{tabular}{lccccc}
\hline & & & \multicolumn{3}{c}{ Collinearity } \\
Variable & Coefficient & $T$ statistic & $P$ & \multicolumn{2}{c}{$\begin{array}{c}\text { stistics } \\
\end{array}$} \\
& & & & Tolerance & VIF \\
\hline$C$ & -0.021 & -0.113 & 0.910 & & \\
$D^{2}$ & -0.076 & -10.310 & 0.000 & 0.121 & 8.298 \\
$J^{2}$ & $-2.030 E-8$ & -2.382 & 0.019 & 0.319 & 3.131 \\
$D J$ & $-9.220 E-5$ & -5.840 & 0.000 & 0.096 & 10.429 \\
\hline
\end{tabular}

$$
\widehat{E}=-0.021-0.076 D^{2}-2.03 \times 10^{-8} J^{2}-9.22 \times 10^{-5} D J .
$$

\subsubsection{Mean Velocity Variation in a Certain Window Width} as an Independent Variable. The velocity variation in a certain window width can be obtained by integrating the acceleration data in a certain window, that is,

$$
S(t, w)=\int_{t-w}^{t} a(t) \mathrm{d} t
$$

where $S(t, w)$ is the velocity variation in a moving window and $w$ is the window width.

Xiao et al. [5] recommended $8 \mathrm{~ms}$ as the optimal $w$. Hence, in our paper, we set $w$ as 8 ms. Notably, $S(t, 8)$ changes with $t$ along the time axis, and thus, it is not unique for a specific acceleration curve. Therefore, $S(t, 8)$ and $E$ cannot have a one-to-one correspondence. Although $S(t, 8)$ can be both positive and negative, the number of negative values are dominant, and the absolute values of the positive and negative numbers are considerably different. Therefore, we considered the mean of $S(t, 8)$ as one of the independent variables (denoted by $\Delta v_{8 \mathrm{~ms}}$ ). Specifically,

$$
\Delta v_{8 \mathrm{~ms}}=\frac{1}{N} \sum_{t} S(t, 8) \text {. }
$$

Note that for each acceleration curve, there exists a corresponding $\Delta v_{8 \mathrm{~ms}}$. Therefore, a one-to-one correspondence can be achieved between the variables. We calculated the $\Delta v_{8 \mathrm{~ms}}$ corresponding to the different acceleration curves associated with $E$ for different $D$. The scatter plot of the three variables is shown in Figure 14.

The least-squares method was adopted to perform multiple regression: $E$ was considered as the dependent variable, and $D$ and $\Delta v_{8 \mathrm{~ms}}$ were used as the independent variables. We implemented the linear regression process in SPSS 16.0. The default 95\% confidence interval was selected. The statistics of the regression model are shown in Table 13, and the coefficients and their significance are shown in Table 14.

Tables 13 and 14 show that the $R$ square of the entire model is 0.575 . All the coefficients are statistically significant at $P<0.05$, except for the constant term.

Furthermore, we adopted a quadratic model for the regression analysis. The expression of the regression model was similar to equation (5), although $\Delta v_{8 \mathrm{~ms}}$ substituted $M$. We input these data into SPSS 16.0. The default 95\% confidence interval was selected. The statistics of the quadratic model are shown in Table 15, and the coefficients and their significances are shown in Table 16.

The comparison of Tables 13 and 15 indicates that the quadratic model is superior to the linear model, with a greater adjusted $R$ square and lower standard error of regression. It can be seen from the above two tables that the $R$ square of the entire model is 0.796 , and the $P$ value of the model is 0 , which is less than $5 \%$ of the significance level, indicating that the model is reliable. The $P$ value corresponding to $D^{2}$ and $D \Delta v_{8 \mathrm{~ms}}$ is 0 , less than $5 \%$ of the significance level, and the absolute value of the $T$ statistic is greater than 2 , indicating significance. However, the $P$ value corresponding to $\Delta v_{8 \mathrm{~ms}}^{2}$ is 0.541 , which is greater than the significance level. The absolute value of the corresponding $T$ statistic of $\Delta v_{8 \mathrm{~ms}}^{2}$ is less than 2 . The $P$ values corresponding to $D$ and $\Delta v_{8 \mathrm{~ms}}$ are 0.664 and 0.08 , respectively, which is greater than the saliency level of $5 \%$, and the absolute value of the corresponding $T$ statistic is less than 2. Therefore, the nonlinear relationship is not significant. Because the tolerance values of $D, \Delta v_{8 \mathrm{~ms}}, D^{2}$, and $\Delta v_{8 \mathrm{~ms}}^{2}$ are less than 0.1 and their VIF values are greater than 10 , significant multicollinearity is considered to occur among the independent variables except for $D \Delta v_{8 \mathrm{~m}}$.

We excluded the variables that were not statistically significant at the $5 \%$ significance level to adjust the intimal regression model. The statistics of the adjusted regression model are shown in Table 17, and the coefficients and their significance are shown in Table 18.

According to the two abovementioned tables, the $R$ square of this model is 0.795 , and the $P$ value of the model is 0 , which is less than $5 \%$ of the significance level, indicating that the model is reliable. The $P$ values corresponding to the independent variables are less than $5 \%$ of the significance level, and the absolute values of the $T$ statistic are greater than 2, which indicates significance. Because the tolerance values of the independent variables are greater than 0.1 and their VIF values are less than 10 , no significant multicollinearity is considered to exist among the independent variables. The adjusted regression equation is expressed as 


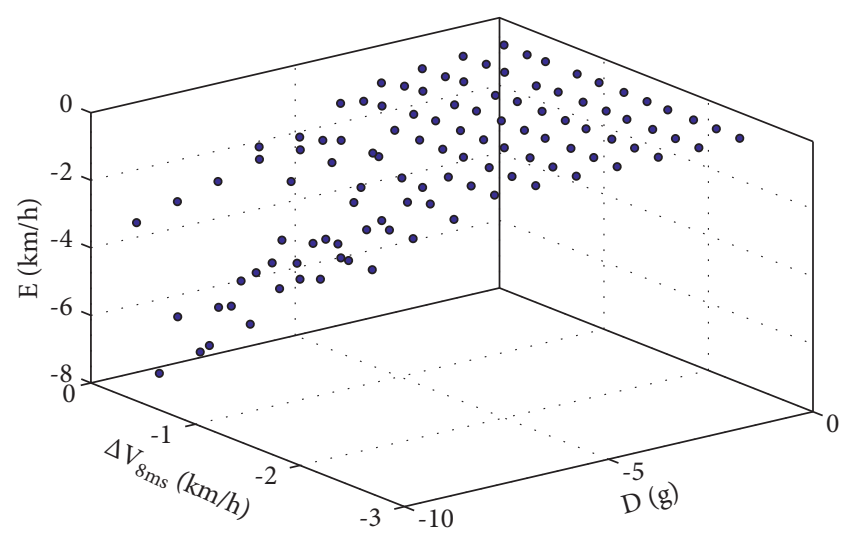

FIGURE 14: Velocity variation error-mean velocity variation in a window width of $8 \mathrm{~ms}$-discrimination threshold relationship diagram.

TABLE 13: Statistics of the linear model (mean velocity variation in a certain window width).

\begin{tabular}{lc}
\hline Name & Value \\
\hline$R$ square & 0.575 \\
Adjusted $R$ square & 0.567 \\
Standard error of regression & 1.028 \\
Sum of residuals & 113.168 \\
$F$ statistic & 72.332 \\
$P$ & 0.000 \\
\hline
\end{tabular}

TABLE 14: Significance of coefficients (mean velocity variation in a certain window width).

\begin{tabular}{lccc}
\hline Variable & Coefficient & $T$ statistic & $P$ \\
\hline$C$ & -0.019 & -0.064 & 0.949 \\
$D$ & 0.370 & 10.829 & 0.000 \\
$\Delta v_{8 \mathrm{~ms}}$ & -0.715 & -5.235 & 0.000 \\
\hline
\end{tabular}

TABLE 15: Statistics of quadratic models (mean velocity variation in a certain window width).

\begin{tabular}{lc}
\hline Name & Value \\
\hline$R$ square & 0.796 \\
Adjusted $R$ square & 0.786 \\
Standard error of regression & 0.72263 \\
Sum of residuals & 54.308 \\
$F$ statistic & 81.144 \\
$P$ & 0.000 \\
\hline
\end{tabular}

equation (12), and its three-dimensional graph is shown in Figure 15.

$$
\widehat{E}=0.616+0.66 \Delta v_{8 \mathrm{~ms}}-0.069 D^{2}+0.25 D \Delta v_{8 \mathrm{~ms}} .
$$

3.2.4. Choice of the Model Equation. To determine the appropriate model to be applied in the ACNS, we considered the following factors.

(1) Fitting degree: This value should be high enough to more accurately reflect the actual speed change loss.
TABLE 16: Significance of coefficients (mean velocity variation in a certain window width).

\begin{tabular}{lccccc}
\hline & & & \multicolumn{3}{c}{$\begin{array}{c}\text { Collinearity } \\
\text { statistics }\end{array}$} \\
Variable & Coefficient & $T$ statistic & $P$ & \multicolumn{3}{c}{ Tolerance } & VIF \\
\hline$C$ & & & & & \\
$D$ & 0.649 & 1.284 & 0.202 & & \\
$\Delta v_{8 \mathrm{~ms}}$ & -0.052 & -0.435 & 0.664 & 0.041 & 24.642 \\
$D^{2}$ & 0.917 & 1.768 & 0.080 & 0.034 & 29.204 \\
$\Delta v_{8 \mathrm{~ms}}^{2}$ & -0.073 & -7.691 & 0.000 & 0.050 & 19.906 \\
$D \Delta v_{8 \mathrm{~ms}}$ & 0.094 & 0.614 & 0.541 & 0.039 & 25.537 \\
\hline
\end{tabular}

TABLE 17: Statistics of regression models (mean velocity variation in a certain window width).

\begin{tabular}{lc}
\hline Name & Value \\
\hline$R$ square & 0.795 \\
Adjusted $R$ square & 0.789 \\
Standard error of regression & 0.71773 \\
Sum of residuals & 54.604 \\
$F$ statistic & 136.903 \\
$P$ & 0.000 \\
\hline
\end{tabular}

TABLE 18: Significance of coefficients of the adjusted model (mean velocity variation in a certain window width).

\begin{tabular}{lccccc}
\hline & & & \multicolumn{3}{c}{ Collinearity } \\
Variable & Coefficient & $T$ statistic & $P$ & \multicolumn{2}{c}{ stics } \\
& & & & Tolerance & VIF \\
\hline$C$ & 0.616 & 2.570 & 0.012 & & \\
$\Delta v_{8 \mathrm{~ms}}$ & 0.660 & 3.480 & 0.001 & 0.252 & 3.962 \\
$D^{2}$ & -0.069 & -15.255 & 0.000 & 0.216 & 4.634 \\
$D \Delta v_{8 \mathrm{~ms}}$ & 0.250 & 8.383 & 0.000 & 0.132 & 7.596 \\
\hline
\end{tabular}

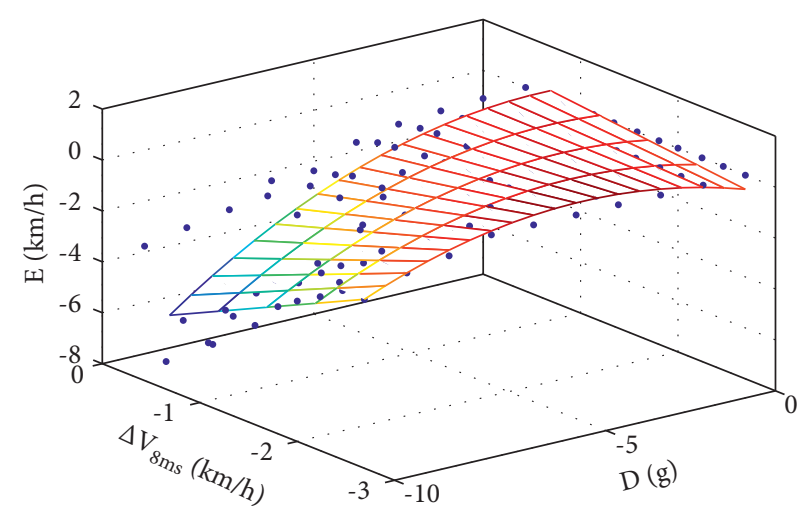

FIGURE 15: Velocity variation error-mean velocity variation in a window width of $8 \mathrm{~ms}$-discrimination threshold regression equation $3 \mathrm{D}$ graph.

(2) Calculation efficiency: Because the ACNS requires a high computing efficiency, the results must be calculated with a high efficiency after the collision. Therefore, a simple and efficient model should be selected.

According to the comparison of the regression models, including $M, J$, and $\Delta v_{8 \mathrm{~ms}}$, equation (12) has the highest $R$ square (that is, the highest fitting degree), while equation (9) 
has the lowest $R$ square (that is, the lowest fitting degree). However, in the case of equation (12), not every $P$ and $T$ statistic corresponding to each item in the equation passes the significance test. Furthermore, although the fitting degree of equation (6) is not the highest, the square terms of the independent variable in the equation exhibit a nonlinear relationship according to the significance test. On the collision curve, $M$ is the point with the largest absolute value, and thus, we can approximately determine the trend of the entire curve according to this point. Compared with $J$ and $\Delta v_{8 \mathrm{~ms}}, M$ can more accurately reflect the shape of the collision curve. Therefore, in this paper, we selected $M$ as the independent variable rather than $J$ and $\Delta v_{8 \mathrm{~ms}}$ and used equation (6) as the correlation model of acceleration, discrimination threshold, and velocity variation error.

The comparison of the three models indicates that the acceleration peak can be promptly obtained, and the calculation speed is very fast. To improve the accuracy of the velocity variation, we can introduce equation (6) in the automatic crash notification (ACN) velocity variation algorithm. Specifically, $\widehat{E}$ obtained from the correlation model is added to the ACN velocity variation calculation result. Hence, we obtain

$$
\Delta v^{\prime}=\Delta v_{\mathrm{ACN}}+\widehat{E},
$$

where $\Delta v^{\prime}$ is the improved velocity variation, and $\widehat{E}$ can be determined according to equation (6).

\section{Design of an ACN Algorithm with a Velocity Variation Correction Function}

Based on the traditional ACN algorithm, this paper proposes an ACN algorithm with the velocity variation correction function (VVCF), which incorporates the established correlation model of $\widehat{E}, D$, and $M$, as shown in Figure 16. First, $D$ needs to be determined. Generally, this value can be set based on uneven driving tests or emergency braking tests. Then, the ACNS collects the acceleration data and determines the acceleration peak $(M)$. Subsequently, the acceleration peak is compared with the discrimination threshold. If the acceleration peak is less than $M$, the system determines that a collision has occurred; however, if the acceleration peak is larger than $M$, the acceleration data continue to be collected and compared with the discrimination threshold. Finally, once the ACNS determines that a collision has occurred, the acceleration-based collision algorithm determines whether it is necessary to seek help and transmit the accident information [30]. If it is not necessary to seek help and transmit the accident information, the algorithm is automatically terminated. Otherwise, the system calculates $\Delta v^{\prime}$ according to equations (6) and (13) and sends it to the rescue centre, along with the other accident information.

\section{Verification of the Algorithm}

5.1. Crash Test. After designing the ACN algorithm with VVCF, it was necessary to verify whether this algorithm can enhance the accuracy of the velocity variation information sent by the ACNS in actual collision accidents through experiments. Therefore, in this paper, we conducted sled tests and real vehicle crash tests to verify the effectiveness and reliability of the algorithm. Notably, the sled test can be carried out many times, and the collision acceleration data of multiple sets of different initial velocities can be obtained. Although the actual car crash test cannot be carried out many times, the results of such tests most closely mimic the actual crash situation, so the verification results of actual car tests are the most convincing. When the sled and real vehicle crash test data are combined, the verification reliability can be improved.

5.1.1. Sled Test. In this paper, the DAPG-QJ-MNPZ sled test system was used for the sled test. To obtain the collision acceleration data of the sled at different initial velocities, sled collision tests were conducted at initial velocities of $30 \mathrm{~km} / \mathrm{h}$, $34 \mathrm{~km} / \mathrm{h}, 50 \mathrm{~km} / \mathrm{h}$, and $56 \mathrm{~km} / \mathrm{h}$. The massive impact force during the collision may cause the sled to vibrate, resulting in a large oscillation in the collected acceleration data in the $x$ direction. To eliminate the interference of the sled vibration on the collected acceleration signals, we used the CFC60 filter to filter the collected acceleration data at a sampling rate of $1000 \mathrm{~Hz}$. Figure 17 shows the crash acceleration curves between the simulation and test results at $34 \mathrm{~km} / \mathrm{h}$, $50 \mathrm{~km} / \mathrm{h}$, and $56 \mathrm{~km} / \mathrm{h}$ after filtering the disturbing acceleration. When the initial speed is $34 \mathrm{~km} / \mathrm{h}$, the tested and simulated sleds reach a peak acceleration of $-20.42 \mathrm{~g}$ and $-20.46 \mathrm{~g}$ at $0.136 \mathrm{~s}$ and $0.141 \mathrm{~s}$, respectively. When the initial speed is $50 \mathrm{~km} / \mathrm{h}$, the tested and simulated sleds reach a peak acceleration of $-36.51 \mathrm{~g}$ and $-34.38 \mathrm{~g}$ at $0.15 \mathrm{~s}$ and $0.16 \mathrm{~s}$, respectively. When the initial speed is $56 \mathrm{~km} / \mathrm{h}$, the tested and simulated sleds reach a peak acceleration of $-42.84 \mathrm{~g}$ and $-50.7 \mathrm{~g}$ at $0.11 \mathrm{~s}$ and $0.12 \mathrm{~s}$, respectively. The simulated and tested curves at these speeds are well matched, in accordance with the comparison shown in Figure 9. Hence, the accuracy of the model is verified.

5.1.2. Real Vehicle Crash Test. To achieve highly convincing test results, $50 \mathrm{~km} / \mathrm{h}$ full-frontal vehicle-to-rigid fixed barrier testing was conducted at the Shanghai Municipal Bureau of Quality and Technical Supervision. The test vehicle was a Proton four-door sedan with a kerb weight of $1308 \mathrm{~kg}$. Figure 18 shows the vehicle before and after the collision.

Data were acquired using the DTS TDAS/G5 acquisition instrument, as shown in Figure 19. The system memory is 16 bits, sensing capacity is $200 \mathrm{~g}$, and sampling rate is $10,000 \mathrm{~Hz}$. The acceleration curve of the full-frontal impact at $50 \mathrm{~km} / \mathrm{h}$ is shown in Figure 20.

According to the figure, three peaks occur in the acceleration curve at $0.13 \mathrm{~s}, 0.15 \mathrm{~s}$, and $0.18 \mathrm{~s}$, consistent with the three-level stiffness distribution at the front of the vehicle. The front bumper, air intake grille, anticollision steel beam, foam material, and engine cover constitute the first-level deformation zone. The material density of these components is generally low, and they obviously cannot absorb much energy. Therefore, the first peak value is small, reaching $-20.36 \mathrm{~g}$. The radiator bracket, suspension system, engine, transmission, 


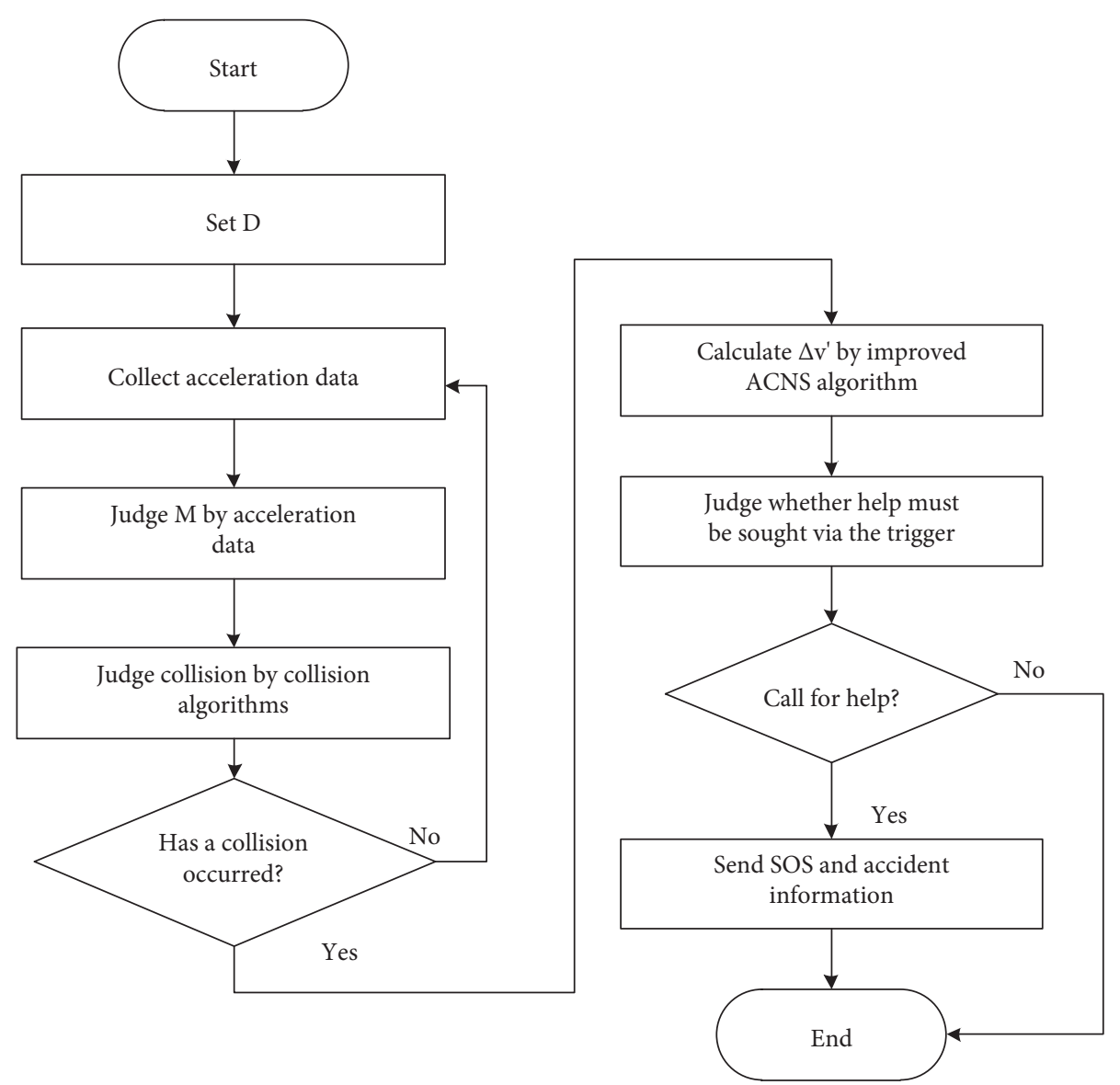

Figure 16: ACN algorithm with VVCF workflow.

longitudinal beam, and frame constitute the second-level compatibility zone, which can provide sufficient rigidity for the vehicle and limit the amount of deformation of the front of the body. In this case, the acceleration peak value reaches $-31.78 \mathrm{~g}$. The steering column, firewall, instrument panel, windshield, A-pillar, and other components constitute the third-level occupant protection zone. The components in this area need to have sufficient rigidity, so the third peak value is greater than the first two peaks, reaching $-38.91 \mathrm{~g}$.

5.2. Validation Analysis. To examine the accuracy enhancement in the velocity variation calculated according to the algorithm with VVCF, the proposed ACN algorithm designed in this paper was imported to an ACNS terminal and compared with the traditional ACN algorithm in terms of the velocity variation outputs. The ACNS terminal consisted of an STM32F103C8T6 microprocessor, an ATK-S1216F8-BD GPS/BD module, an ATK-SIM800C module, a controller area network (CAN) communication interface, and an NRF2401 wireless radio frequency (RF) module, as shown in Figure 21. The whole system was based on an STM32F103C8T6 microprocessor. A CAN communication interface was used to receive the information from the vehicle. The NRF2401 wireless RF module could receive the acceleration data transmitted by the accelerometers as a wireless signal. The ATK-S1216F8-BD GPS/BD module could receive and parse the location information. All the integrated information was delivered to the emergency management system (EMS) by the ATKSIM800C module. The whole ACNS terminal is shown in Figure 22. To facilitate verification, we used a mobile phone to represent the EMS as the receiving end of the accident information.

5.2.1. Discrimination Threshold Setting and Anti-FalseAlarm Ability of the ACNS. The discrimination threshold, which distinguishes collisions from uneven road driving, emergency braking, and so on, is a critical setting to avoid false alarms. Table 19 shows the peak acceleration of the vehicle body (denoted by $a_{x p}$ ) under the typical working conditions at different speeds [31].

According to Table 19, when a vehicle drives over flat roads or passes through ordinary barricades, a relatively small absolute value of the discrimination threshold can satisfy the requirements of resisting the road interference. However, for certain roads, a larger discrimination threshold is needed.

In our ACNS terminal, we set the discrimination threshold as $-9 g$. This value is larger than the peak acceleration of the vehicle body under most circumstances. Consequently, the occurrence possibility of false alarms is low. 


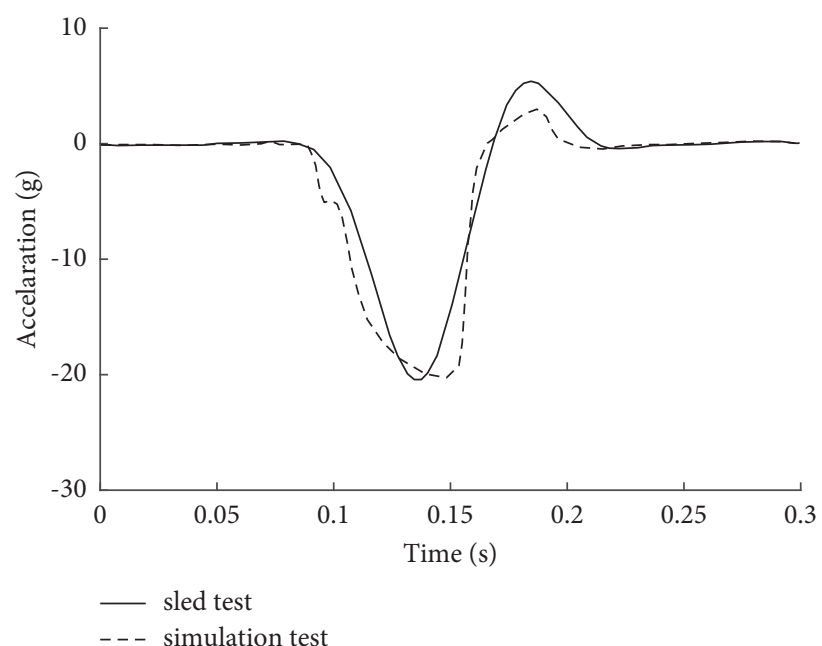

(a)

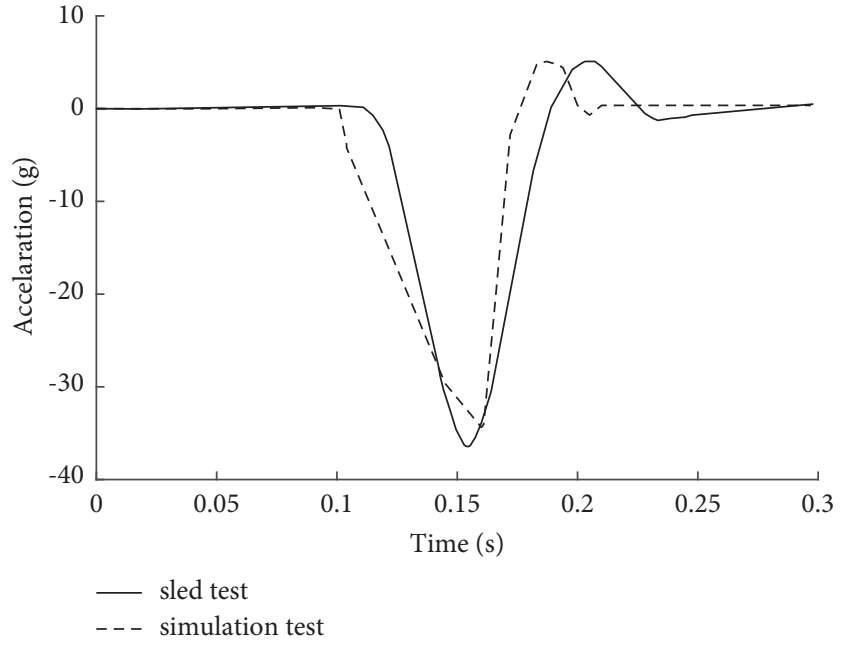

(b)

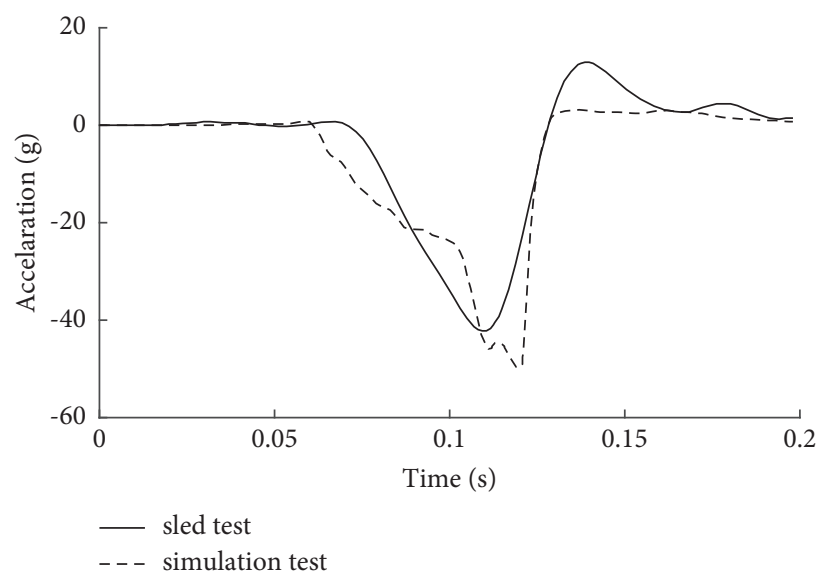

(c)

Figure 17: Collision acceleration curve.(a) Acceleration curve of the simulation and real sled crash test after filtering at $34 \mathrm{~km} / \mathrm{h}$. (b) Acceleration curve of the simulation and real sled crash test after filtering at $50 \mathrm{~km} / \mathrm{h}$. (c) Acceleration curve of the simulation and real sled crash test after filtering at $56 \mathrm{~km} / \mathrm{h}$.

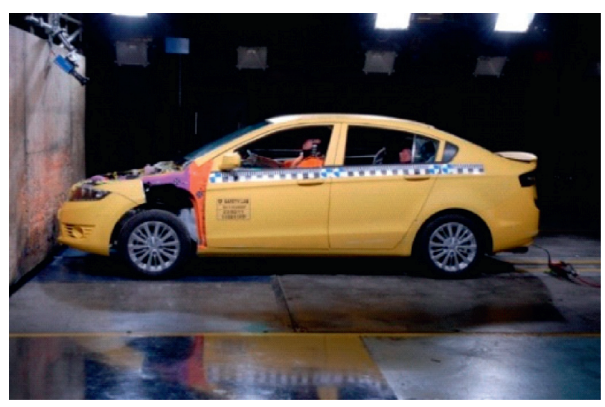

(a)

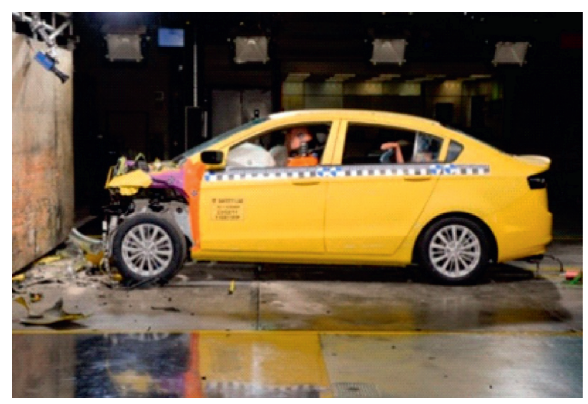

(b)

Figure 18: Test vehicle before and after the collision. (a) Before vehicle collision. (b) After vehicle collision.

5.2.2. Evaluation of the ACN Algorithm with VVCF. According to the European regulation ECE R94, when a car frontally impacts a fixed rigid barrier at a speed of more than $30 \mathrm{~km} / \mathrm{h}$, the air bag should be deployed, which means that the ACNS should be triggered. Hence, we considered the collision acceleration data of the sled at the initial speed of
$30 \mathrm{~km} / \mathrm{h}$ to perform the verification experiment. The traditional ACN algorithm was based on the moving window integral algorithm (MWIA) [5]. We used the Microcontroller Development Kit (MDK) Version 5 to programme the algorithm. The sets of acceleration data and discrimination threshold were directly written into the 


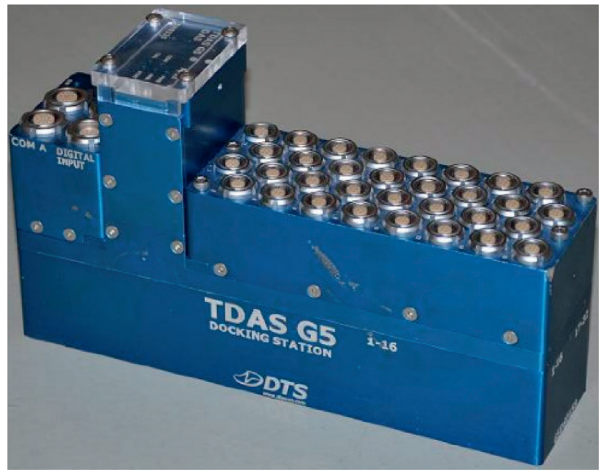

Figure 19: Acquisition instrument.

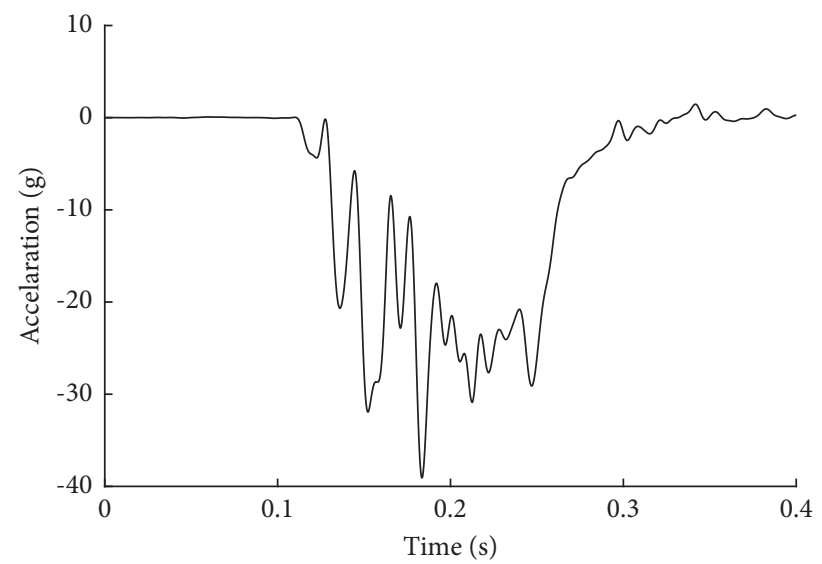

Figure 20: Acceleration curve of the real vehicle crash test.

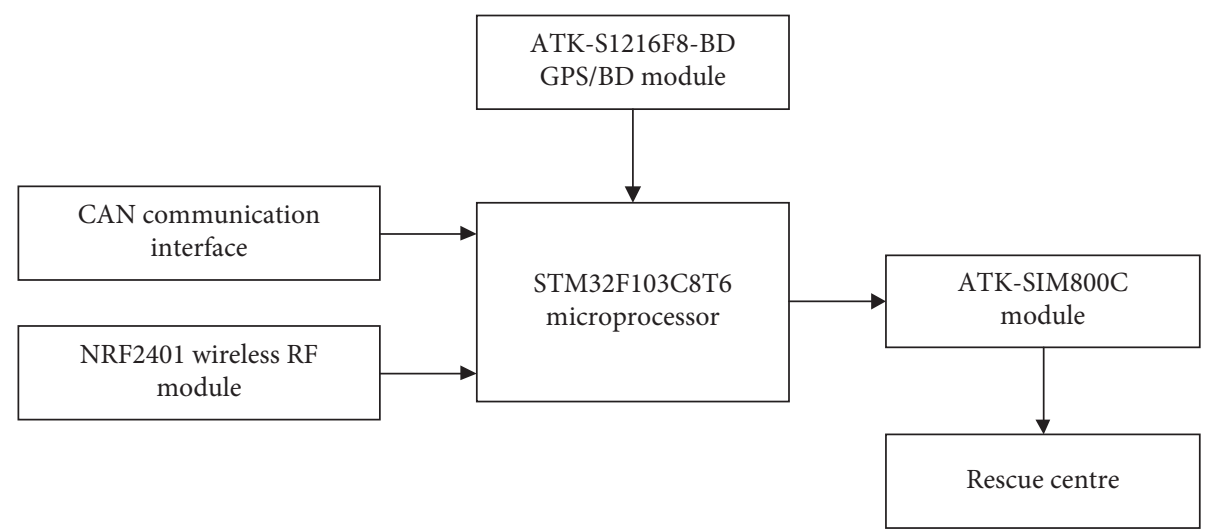

FIgURE 21: Diagram of the ACNS terminal.

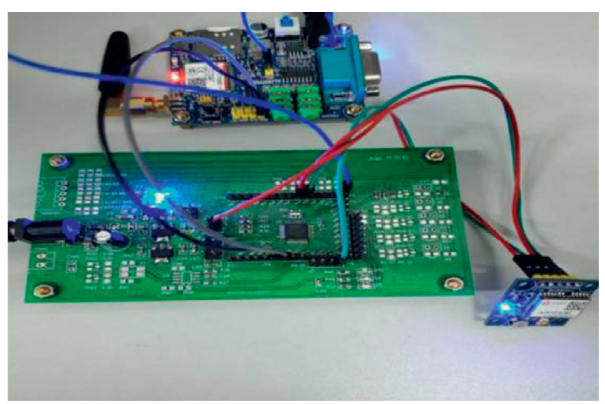

FIGURE 22: ACNS terminal. 
TABle 19: Peak acceleration under typical working conditions.

\begin{tabular}{lcc}
\hline Working conditions & $\begin{array}{c}\text { Speed } \\
(\mathrm{km} / \mathrm{h})\end{array}$ & $a_{x p}(\mathrm{~g})$ \\
\hline Driving on gravel road & 60 & -1 to -0.8 \\
Driving on washboard road & 60 & -5 to -3 \\
$\begin{array}{l}\text { Driving on stone road } \\
\text { Emergency braking }\end{array}$ & 50 & -2 to -1.5 \\
$\begin{array}{l}\text { Passing through } 110 \mathrm{~mm} \text { high } \\
\text { barricades }\end{array}$ & 60 & -1 to -0.8 \\
\hline
\end{tabular}

program. Then, this program was imported to the ACNS terminal. To facilitate comparison with the traditional algorithm, the ACN algorithm with VVCF was also based on the MWIA, except that a small section of the speed change correction programme was introduced. In addition, the same acceleration data and discrimination threshold were written into the program. The information received by the mobile phone is shown in Tables 20 and 21 .

According to Tables 20 and 21, even though the acceleration data and the discrimination threshold are the same, the velocity variations calculated by the two $\mathrm{ACN}$ algorithms are different. The velocity variation obtained via the traditional algorithm and algorithm with VVCF is $-19.0717 \mathrm{~km} / \mathrm{h}$ and $-22.5088 \mathrm{~km} / \mathrm{h}$, respectively. Ideally, $\Delta v$ should be $30 \mathrm{~km} / \mathrm{h}$ since the sled initial speed is $30 \mathrm{~km} / \mathrm{h}$. Because frictional resistance exists on the sled track, some loss in the impact speed is expected. By examining the acceleration data, we determined $t_{0}$ and $t_{\mathrm{p}}$ and integrated the acceleration data with an initial speed of $30 \mathrm{~km} / \mathrm{h}$. Consequently, the actual velocity variation during the collision is calculated to be $-23.2599 \mathrm{~km} / \mathrm{h}$. Therefore, the result obtained via the algorithm with VVCF is closer to the actual value.

\section{Discussion}

6.1. Sensitivity Analysis. To investigate the impact of the discrimination threshold and collision speed on the efficiency of the proposed ACN algorithm with VVCF, the acceleration data under different discrimination thresholds and initial velocities were imported into the two programs to calculate the respective velocities. The discrimination threshold was set to different values ranging between $-10 \mathrm{~g}$ and $0 \mathrm{~g}$. Acceleration data at initial velocities of $30 \mathrm{~km} / \mathrm{h}, 34 \mathrm{~km} / \mathrm{h}, 50 \mathrm{~km} / \mathrm{h}$, and $56 \mathrm{~km} / \mathrm{h}$, obtained by sled tests, and $50 \mathrm{~km} / \mathrm{h}$, obtained by the real vehicle crash test, were directly written into the ACNS terminal. The results are shown in Tables 22 and 23. The third row of Table 22 represents the initial velocity; the first column represents the discrimination thresholds; and the data in the remaining table represent $E$. Similarly, the third row of Table 23 represents the initial velocity corresponding to the collision acceleration data. The first column represents the discrimination threshold.

Let $E^{\prime}$ denote the errors between the actual value and velocity variation calculated using the $\mathrm{ACN}$ algorithm with VVCF, that is,

$$
E^{\prime}=\Delta v-\Delta v^{\prime}
$$

The remaining data in Table 23 indicate the results.
TABLE 20: Output of the traditional ACN algorithm.

\begin{tabular}{lc}
\hline Whether a collision occurred & Yes \\
\hline Longitude & $119.52185 \mathrm{E}$ \\
Latitude & $32.20446 \mathrm{~N}$ \\
UTC date & $2020 / 11 / 16$ \\
UTC time & $12: 02: 12$ \\
Velocity variation in collision & $-19.0717 \mathrm{~km} / \mathrm{h}$ \\
\hline
\end{tabular}

TABLE 21: Output of the ACN algorithm with VVCF.

\begin{tabular}{lc}
\hline Whether a collision occurred & Yes \\
\hline Longitude & $119.52185 \mathrm{E}$ \\
Latitude & $32.20446 \mathrm{~N}$ \\
UTC date & $2020 / 11 / 16$ \\
UTC time & $13: 19: 32$ \\
Velocity variation in collision & $-22.5088 \mathrm{~km} / \mathrm{h}$ \\
\hline
\end{tabular}

TABLE 22: Errors in the speed changes obtained via the traditional ACN algorithm.

\begin{tabular}{lccccc}
\hline \multicolumn{5}{c}{$E(\mathrm{~km} / \mathrm{h})$} \\
$D(\mathrm{~g})$ & \multicolumn{5}{c}{ Initial speed $v_{0}(\mathrm{~km} / \mathrm{h})$} \\
& 30 & 34 & 50 & 56 & $50^{*}$ \\
\hline-6 & 2.27 & 1.46 & 0.60 & 0.58 & 1.75 \\
-7 & 2.74 & 1.70 & 0.85 & 0.58 & 1.82 \\
-8 & 3.56 & 2.25 & 1.14 & 0.85 & 1.87 \\
-9 & 4.19 & 2.88 & 1.47 & 1.18 & 1.97 \\
-10 & 4.87 & 3.23 & 1.83 & 1.55 & 2.04 \\
\hline
\end{tabular}

* means the real vehicle test.

TABLE 23: Errors in the speed changes obtained using the ACN algorithm with VVCF.

$$
E^{\prime}(\mathrm{km} / \mathrm{h})
$$

$D(\mathrm{~g}) \quad$ Initial speed $v_{0}(\mathrm{~km} / \mathrm{h})$

\begin{tabular}{lccccc} 
& 30 & 34 & 50 & 56 & $50^{*}$ \\
\hline-6 & 1.19 & 0.74 & 0.53 & 0.26 & 1.58 \\
-7 & 1.00 & 0.40 & 0.55 & 0.55 & 1.65 \\
-8 & 1.04 & 0.24 & 0.34 & 0.46 & 1.23 \\
-9 & 0.75 & 0.03 & 0.04 & 0.24 & 0.73 \\
-10 & 0.38 & 0.59 & 0.36 & 0.07 & 0.07
\end{tabular}

${ }^{*}$ means the real vehicle test.

According to Tables 22 and 23, when the initial velocity of the vehicle collision is low, the calculation error of the traditional ACN algorithm is large, and the error of the ACN algorithm with VVCF is significantly reduced. When the initial velocity of the vehicle collision is high, the errors in the velocity variation calculated via the traditional ACN algorithm and ACN algorithm with VVCF are small, and the latter value is reduced. Because the original error is not large, the improvement is not very obvious. This phenomenon occurs because the calculation error of the traditional ACN algorithm decreases as the initial velocity of the vehicle collision increases (according to equation (6), a smaller $M$ corresponds to a larger initial collision speed). Hence, the proposed ACN algorithm with VVCF exhibits a higher performance when the vehicle is at a low speed by providing 


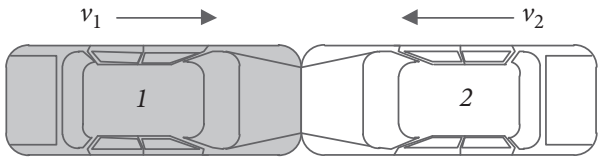

(a)

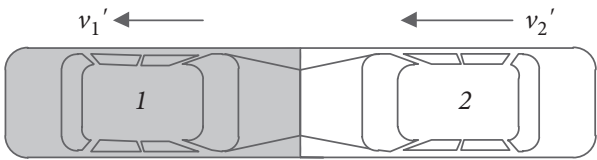

(b)

Figure 23: Head-to-head collision. (a) Before head-to-head collision. (b) After head-to-head collision.

larger error compensation. Moreover, the algorithm attains a certain improvement in the calculation of the velocity variation information for a vehicle at high speed.

6.2. Head-to-Head Collision Analysis. The ratio of the vehicle speed after a crash (denoted by $v^{\prime}$ ) to the speed before the vehicle collision $v_{0}$ is known as the coefficient of restitution and can be expressed as

$$
e=-\frac{v^{\prime}}{v_{0}},
$$

When $e=1$, the collision is elastic; when $0<e<1$, the collision is inelastic; and when $e=0$, the collision is completely inelastic [32].

The regression models adopted in this paper were based on the data generated by sled tests. The considered collisions can be regarded as completely inelastic collisions since the sled and rigid barriers are affixed. In the case of real vehicle collisions, when the vehicles impact the barrier at medium and low speeds, the collision can be considered an inelastic collision, for which $0<e<1$. However, when the collision speed exceeds a certain limit value, the collision is considered completely inelastic. For instance, when the collision speed is $50 \mathrm{~km} / \mathrm{h}$, the coefficient of restitution $e$ is 0.1 , and the collision can be considered to be completely inelastic. Hence, our method is mainly suitable for vehicle-to-rigid barrier frontal collisions at high initial velocities.

In the real world, head-to-head collisions between two vehicles occur in certain scenarios. The characteristics of such collisions are completely different from those of a frontal crash with a fixed object. Figure 23 shows the vehicles before and after head-to-head collisions.

The significant difference between head-to-head collisions and vehicle-to-rigid barrier frontal collisions pertains to the coefficient of restitution. The coefficient of restitution for the former case is

$$
e=-\frac{v_{1}^{\prime}-v_{2}^{\prime}}{v_{1}-v_{2}}
$$

where $v_{1}$ and $v_{2}$ are the initial impact speeds of the two vehicles, and $v_{1}^{\prime}$ and $v_{2}^{\prime}$ are the post-crash speeds of the two vehicles. It is difficult to judge whether $e$ equals 0 under this circumstance.

To investigate the applicability of our algorithm in the case of a head-to-head collision, we introduce the equivalent barrier velocity (EBV), as shown in Figure 24.

The equivalent barrier velocity for vehicle 1 , denoted by $v_{B 1}$, is expressed as

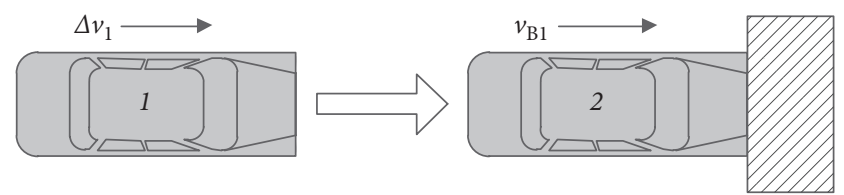

FIgURE 24: Velocity variation and equivalent barrier velocity.

$$
v_{B 1}=\left|v_{2}-v_{1}\right| \sqrt{\frac{k_{2}}{k_{1}+k_{2}} \frac{m_{2}}{m_{1}+m_{2}}},
$$

where $k_{1}$ and $k_{2}$ are the stiffnesses of vehicles 1 and 2, respectively, and $m_{1}$ and $m_{2}$ are the masses of vehicles 1 and 2, respectively [33].

According to equations (16) and (17), we can derive the following conclusions.

(1) When $e=0$, the stiffnesses of the unit masses of vehicles 1 and 2 are equal; that is, $\left(k_{1} / m_{1}=k_{2} / m_{2}\right)$, $v_{B 1}=\Delta v_{1}$.

(2) When $v_{1}=-v_{2}, m_{1}=m_{2}$, and $k_{1}=k_{2}, v_{B 1}=\left|v_{1}\right|$.

(3) In other cases, it is impossible to directly suggest the connection among $v_{B 1}, \Delta v_{1}$, and $v_{1}$.

Note that $\Delta v^{\prime}$ calculated by the ACN algorithm with VVCF is approximately equal to $v_{B 1}$. Therefore, the correction procedure for the velocity variation in the head-tohead-collision vehicle can be summarized as follows:

(1) Calculate $\Delta v^{\prime}$ by using the ACN algorithm with VVCF. Let $v_{B 1}$ be $\Delta v^{\prime}$.

(2) If $k_{1} / m_{1}=k_{2} / m_{2}$ and vehicles 1 and 2 are affixed, $\Delta v_{1}=v_{B 1}$.

(3) If the two vehicles are similar and collide with each other at similar initial speeds, $\left|v_{1}\right|=v_{B 1}$.

(4) In other cases, we cannot definitively obtain the correct $\Delta v_{1}$ or $v_{1}$.

\section{Conclusion}

The ACNS velocity variation algorithm was enhanced in this paper by establishing the correlation model between $E$ and the influencing factors, thereby rendering the calculated velocity variation more accurate and increasing the rescue efficiency. First, we analysed the factors affecting the accuracy of velocity variation based on the acceleration data generated by sled tests. Then, the correlation between the accuracy of the velocity variation and influencing factors was modelled. Next, we proposed an ACNS velocity variation algorithm based on this correlation model. The reliability of the new algorithm was 
verified over an ACNS terminal. The results showed that when the program of the ACNS terminal contains the ACN algorithm with VVCF, the error between the calculated and actual velocity variations is reduced, which indicates that the improved ACNS velocity variation algorithm proposed in this paper is effective. By introducing the EBV, we investigated the applicability of our algorithm in the case of a head-to-head collision. The results indicated that under certain conditions, the EBV is equal to the velocity variation or initial collision speed.

Future work can be aimed at extending this research in several directions. First, the collision angle can be considered. In this study, the simulation model, sled test, and real vehicle crash test designed in this paper pertained to $100 \%$ frontal overlap rigid barrier collision tests. The correlation model was based on these impact data. However, in actual collision scenarios, rear and side collisions also occur. Thus, it is necessary to verify whether the velocity variation algorithm is suitable for these situations. Second, the impact of the vehicle features on the characteristics of the acceleration curves can be examined. This study was conducted using a simulation model to obtain the correlation model between the velocity variation errors and influencing factors rather than using real collision data. Hence, other factors, such as the characteristics of vehicles and rollovers, should also be considered in future studies.

\section{Nomenclature}

\begin{tabular}{|c|c|}
\hline$\Delta v:$ & Velocity variation \\
\hline$\Delta v_{\mathrm{ACN}}:$ & $\begin{array}{l}\text { Velocity variation calculated via the ACN } \\
\text { algorithm }\end{array}$ \\
\hline$t_{0}:$ & Starting time of collision \\
\hline$t_{\mathrm{P}}:$ & Ending time of collision \\
\hline$t:$ & Current moment \\
\hline$a(t):$ & $\begin{array}{l}\text { Acceleration corresponding to the current } \\
\text { moment }\end{array}$ \\
\hline$t_{d t}:$ & $\begin{array}{l}\text { Starting time of collision, as determined by the } \\
\text { ACNS }\end{array}$ \\
\hline$E:$ & Velocity variation error \\
\hline$D:$ & Discrimination threshold \\
\hline M: & Acceleration peak \\
\hline$b_{1}, \ldots, b_{6}:$ & Coefficient of the regression equation \\
\hline$\varepsilon:$ & Regression error \\
\hline$E:$ & Estimated value of $E$ \\
\hline$J(t):$ & Acceleration slope \\
\hline$J:$ & $\begin{array}{l}\text { Mean of the absolute values of the acceleration } \\
\text { slope }\end{array}$ \\
\hline$\Delta t:$ & Time interval between two acceleration signals \\
\hline$a(t-\Delta t):$ & $\begin{array}{l}\text { The acceleration of the previous time relative to } \\
\text { the current moment }\end{array}$ \\
\hline$N:$ & Number of sample points in the time domain \\
\hline$S(t, w)$ & Velocity variation in a moving window. \\
\hline$w:$ & Window width \\
\hline$\Delta v_{8 \mathrm{~ms}}:$ & Mean of the velocity variation in $8 \mathrm{~ms}$ \\
\hline$\Delta v^{\prime}:$ & Improved velocity variation \\
\hline$v_{0}:$ & Initial speed \\
\hline$E^{\prime}:$ & $\begin{array}{l}\text { Errors between the actual velocity variation and } \\
\text { that calculated via the ACN algorithm with } \\
\text { VVCF }\end{array}$ \\
\hline
\end{tabular}

$v^{\prime}: \quad$ Vehicle speed after crash

$e: \quad$ Coefficient of restitution

$v_{1}, v_{2}$ : Initial impact speeds of vehicles 1 and 2, respectively

$v_{1^{\prime}}, v_{2^{\prime}}$ : Post-crash speeds of vehicles 1 and 2, respectively

$v_{B 1}$ : $\quad$ Equivalent barrier velocity for vehicle 1

$k_{1}, k_{2}$ : $\quad$ Stiffness of vehicles 1 and 2 , respectively

$m_{1}, m_{2}$ : Mass of vehicles 1 and 2, respectively.

\section{Data Availability}

The data used to support the findings of this study are available from the corresponding author upon request.

\section{Conflicts of Interest}

The authors declare that they have no conflicts of interest.

\section{Acknowledgments}

This work was supported by the Natural Science Foundation of China (Grant no. 51605197), National Key Research and Development Program of China (Grant no. 2019YFB1600500), Natural Science Foundation of Jiangsu Province (Grant no. BK20160524), Key Research and Development Plan of Zhenjiang City (Grant no. SH2019054), and Jiangsu Government Scholarship for Overseas Studies.

\section{References}

[1] E. Jeong, C. Oh, and J. Lee, "Evaluation of safety benefits of automatic crash information notification systems on freeways," International Journal of Automotive Technology, vol. 15 , no. 3, pp. 495-503, 2014.

[2] J. A. Lahausse, B. N. Fildes, Y. Page, and M. P. Fitzharris, "The potential for automatic crash notification systems to reduce road fatalities," Annals of advances in automotive medicine. Association for the Advancement of Automotive Medicine. Annual Scientific Conference, vol. 52, pp. 85-92, 2008.

[3] G. Ponte, G. A. Ryan, and R. W. G. Anderson, "An estimate of the effectiveness of an in-vehicle automatic collision notification system in reducing road crash fatalities in South Australia," Traffic Injury Prevention, vol. 17, no. 3, pp. 258-263, 2016.

[4] American College of Emergency Physicians, Advanced Automatic Crash Notification and Intelligent Transportation Systems: Implications for the Emergency Physician, Policy Resource and Education Paper (PREP), Irving, TX, USA, 2016.

[5] W. Xiao, Z. Li, W. Chen, Y. Lu, and H. Jiang, "Trigger algorithm of vehicle automatic crash notification system," International Journal of Automotive Technology, vol. 17, no. 2, pp. 273-280, 2016.

[6] F. Y. Jia, "Research on a new generation automatic alarm system of emergency rescue in vehicle accidents," Doctoral Dissertation, Tongji University, Shanghai, China, 2015.

[7] R. H. Ge, W. T. Zhu, and L. Zang, "A study on the control strategy for vehicle impact identification based on improved moving window algorithm," Automotive Engineering, vol. 33, no. 7, pp. 590-593, 2011. 
[8] L. Nassar, M. S. Kamel, and F. Karray, "VANET IR-CAS for safety ACN: information retrieval context aware system for VANET automatic crash notification safety application," International Journal of Intelligent Transportation Systems Research, vol. 14, no. 3, pp. 127-138, 2016.

[9] H. R. Champion and B. Cushing, "Emerging technology for vehicular safety and emergency response to roadway crashes," Surgical Clinics of North America, vol. 79, no. 6, pp. 12291240, 1999.

[10] H. R. Champion, J. Augenstein, A. J. Blatt, B. Cushing, and M. C. Flanigan, "Automatic crash notification and the urgency algorithm: its history, value, and use," Advanced Emergency Nursing Journal, vol. 26, no. 2, pp. 143-156, 2004.

[11] S. L. Schoell, A. N. Doud, A. A. Weaver et al., "Characterization of the occult nature of injury for frequently occurring motor vehicle crash injuries," Accident Analysis \& Prevention, vol. 98, pp. 149-156, 2017.

[12] D. W. Kononen, C. A. C. Flannagan, and S. C. Wang, "Identification and validation of a logistic regression model for predicting serious injuries associated with motor vehicle crashes," Accident Analysis \& Prevention, vol. 43, no. 1, pp. 112-122, 2011.

[13] S. Yoshida, T. Hasegawa, S. Tominaga, and T. Nishimoto, "Development of injury prediction models for advanced automatic collision notification based on Japanese accident data," International Journal of Crashworthiness, vol. 21, no. 2, pp. 112-119, 2016.

[14] J. D. Stitzel, A. A. Weaver, J. W. Talton et al., "An injury severity-, time sensitivity-, and predictability-based advanced automatic crash notification algorithm improves motor vehicle crash occupant triage," Journal of the American College of Surgeons, vol. 222, no. 6, pp. 1211-1219, 2016.

[15] C. Pal, S. Hirayama, S. Narahari et al., "Improvement of injury severity prediction (ISP) of AACN during on-site triage using vehicle deformation pattern for car-to-car (C2C) side impacts," Traffic Injury Prevention, vol. 19, pp. S201-S204, 2018.

[16] Y. Lu, Y. Z. Yin, and H. Y. Ye, "The Influence of threshold of advanced automatic crash notification system on prediction of driver's injury," Automobile Technology, no. 3, pp. 5-9, 2020.

[17] Z. W. Guan, S. H. Wang, W. Q. Liang, M. Zheng, and L. Wu, "The analysis of the key problems about traffic accident speed identification based on braking traces," Advanced Materials Research, vol. 756-759, pp. 4752-4757, 2013.

[18] P. Kubiak, K. Siczek, A. Dąbrowski, and A. Szosland, "New high precision method for determining vehicle crash velocity based on measurements of body deformation," International Journal of Crashworthiness, vol. 21, no. 6, pp. 1-10, 2016.

[19] X. L. Yang, P. Li, T. Lv, and X. H. Liao, "Traffic accident reconstruction technology research," Advanced Materials Research, vol. 756-759, pp. 946-951, 2013.

[20] Q. Lv, "Research and design of auxiliary systems accident identified," Master Thesis, Electronic Science and Technology University, Chengdu, China, 2014.

[21] H. L. Cui, Z. Y. Wang, Q. W. She, and X. Li, "Velocity analysis of vehicle cracking accident," Journal of Xi'an Highway University, vol. 23, no. 1, pp. 84-86, 2003.

[22] J. W. Hu, M. P. Reed, J. D. Rupp, K. Fischer, and A. Adler, "Optimizing seat belt and airbag designs for rear seat occupant protection in frontal crashes," Stapp Car Crash Journal, vol. 61, pp. 67-100, 2017.

[23] Y. Han, X. Li, H. Huang, A. Cang, and K. Mizuno, "A study on the effects of sled acceleration waveform on the injury risk of 3-year-old child occupant," Automotive Engineering, vol. 39, no. 6, pp. 661-666, 2017.

[24] GB 14166-2013, Chinese National Standard for Safety-Belts, Restraint Systems, Child Restraint Systems, and ISOFIX Child Restraint Systems for Occupants of Power-Driven Vehicle, Standards Press of China, Beijing, China, 2013.

[25] Z. X. Li, J. Shen, Y. Lu, and W. Xiao, "Study on trigger algorithm of automatic crash notification system based on power density," Science Technology and Engineering, vol. 15, no. 26, pp. 84-88, 2015.

[26] D. S. Dima and D. Covaciu, "Solutions for acceleration measurement in vehicle crash tests," IOP Conference Series: Materials Science and Engineering, vol. 252, Article ID 012007, 2017.

[27] B. Bhardwaj, R. Bridgelall, L. Chia, P. Lu, and N. Dhingra, "Signal filter cut-off frequency determination to enhance the accuracy of rail track irregularity detection and localization," IEEE Sensors Journal, vol. 20, no. 3, pp. 1393-1399, 2019.

[28] C. H. Koh, K. H. Low, Y. Zhao et al., "Weight threshold estimation of falling UAVs (Unmanned Aerial Vehicles) based on impact energy," Transportation Research Part C: Emerging Technologies, vol. 93, no. 8, pp. 228-255, 2018.

[29] Z. Wei, K. G. Robbersmyr, and H. R. Karimi, "Data-based modeling and estimation of vehicle crash processes in frontal fixed-barrier crashes," Journal of the Franklin Institute, vol. 354, no. 12, pp. 4896-4912, 2017.

[30] A. S. Liu, "Study on trigger decision technology for vehicle accident automatic call system," Master Thesis, Jiangsu University, Zhenjiang, China, 2017.

[31] T. Yang, W. Zheng, J. Zhang, and S. Huang, "Ignition circuit for automotive airbag system," Journal of Tsinghua University, vol. 42 , no. 4 , pp. 527-530, 2002.

[32] P. Kubiak, A. Krzemieniewski, K. Lisiecki, J. Senko, and A. Szosland, "Precise method of vehicle velocity determination basing on measurements of car body deformation-nonlinear method for "full size" vehicle class," International Journal of Crashworthiness, vol. 23, no. 3, pp. 302-310, 2018.

[33] J. Singh, "A fundamental reconsideration of the CRASH3 damage analysis algorithm: the case against uniform ubiquitous linearity between BEV, peak collision force magnitude, and residual damage depth," Traffic Injury Prevention, vol. 14, no. 7, pp. 718-724, 2013. 